Article

\title{
Monitoring of MV Cable Screens, Cable Joints and Earthing Systems Using Cable Screen Current Measurements
}

\author{
Krzysztof Lowczowski ${ }^{1, *} *$, Jozef Lorenc ${ }^{1}$, Andrzej Tomczewski ${ }^{2}$, Zbigniew Nadolny ${ }^{1}(\mathbb{C}$ and \\ Jozef Zawodniak ${ }^{3}$ \\ 1 Institute of Electric Power Engineering, Poznan University of Technology, Piotrowo 3A, \\ 60965 Poznan, Poland; jozef.lorenc@put.poznan.pl (J.L.); zbigniew.nadolny@put.poznan.pl (Z.N.) \\ 2 Institute of Electrical Engineering and Electronics, Poznan University of Technology, Piotrowo 3A, \\ 60965 Poznan, Poland; andrzej.tomczewski@put.poznan.pl \\ 3 Department of Network Asset Management, Enea Operator, Strzeszynska 58, 60479 Poznan, Poland; \\ jj.zawodniak@wp.pl \\ * Correspondence: krzysztof.lowczowski@put.poznan.pl
}

Received: 16 May 2020; Accepted: 30 June 2020; Published: 3 July 2020

\begin{abstract}
The paper presents the possibility of using cable screen earthing current measurements for MV, single-core cable line monitoring. Cable screen earthing current measurement allows the condition of cable screen connections, degradation of earthing systems, and the earthing system integrity to be observed, which allows a condition based maintenance (CBM) strategy to be introduced for cable screens and earthing systems in MV networks. CBM allows the workload and failure rate to be reduced. Analysis of the condition of the cable screen and earthing system is carried out based on the cable screen earthing current trends and rapid changes of the current. The proposed system is integrated with a state of the art advanced metering infrastructure (AMI) and uses AMI data to calculate currents flowing through cable segments of the analyzed feeder. Additionally, a system which counts thermo-mechanical stresses associated with high current incidents in cable screens is proposed. The stresses are assessed using data from conventional protection relays and the ratio of currents in the cable core and cable screens. The stresses can be used as an indicator of the cable joint condition. The presented phenomena are studied carefully in PowerFactory software. Theoretical considerations are confirmed using measurements taken in the real MV cable line. Finally, the results of a SWOT analysis are presented and future research activities are outlined.
\end{abstract}

Keywords: earthing/grounding system; cable screen; quasi-dynamic simulation; advanced metering infrastructure (AMI); cable joint

\section{Introduction}

Cable lines are very important elements of a power system which connect energy sources with electrical loads. Cable lines are reliable since, contrary to overhead lines, they are buried underground and therefore naturally screened from atmospheric conditions. Because of the high reliability of cable lines, the share of the cables in power systems is increasing systematically. The reliability of the distribution system network is very important since loads connected to the distribution network consume approximately $77 \%$ of energy [1]. Unfortunately, cable line failures are difficult to repair since failures are not visible to the naked eye. In order to find the fault location, special tools have to be used. One has to however note that utilization of new technologies allows the condition of cable lines to be monitored and greatly minimizes the risk of failure. Moreover, special tools are able to detect pre-fault conditions, which allows unplanned, prolonged, power interruptions to be avoided, as well as the risk 
of hazardous situations connected with a failure to be reduced, i.e., risk of electrocution in the case of a phase to earth fault. The integral part of cable lines are earthing systems. Earthing systems have been researched since the beginning of power system development up to this day. Research about earthing systems is focused on lattice earth electrodes of high $(>110 \mathrm{kV})$ voltage substations [2-5], optimization of earthing systems construction and monitoring of earthing systems in depths of the network and inside substations [6-9]. Earthing systems ensure the safety of power system operation, i.e., directing lightning strikes to the ground, ensuring the safety of humans under fault conditions, and allowing for voltage measurement $[10,11]$. An earthing system should be characterized by [12]:

- high conductivity,

- robustness against fault current flow,

- long lifespan,

- low resistance and impedance,

- equipotentialisation connections,

- resistivity against corrosion,

- mechanical and electrical durability.

Monitoring of earthing systems is a big challenge because of the large number of earthing systems, i.e., the four biggest polish distribution system operators (DSOs), which operate in almost 100\% of the area of Poland, have 256 thousands substations and all the substations are equipped with earthing systems [13]. Commercial systems for Medium Voltage (MV) earthing system monitoring are able to monitor one earthing system or a few earthing systems, but only in close proximity $[14,15]$. An alternative for conventional monitoring of the earthing systems is to measure and analyze residual cable screen earthing system current- $I_{r c s}$. Utilization of $I_{r c s}$ allows for simultaneous monitoring of many earthing systems in remote locations, and what is most important is that the proposed system utilizes exactly the same hardware as the system for detection and location of earth faults [16-18]. It is possible to detect degradation, mechanical damage, or theft of earthing systems. A system for monitoring of earthing systems allows a condition based maintenance strategy to be introduced, which allows the effectiveness of electric utilities to be improved [19]. This improvement is achieved through reduced numbers of failures and reduced number of maintenance activities connected with reduced operational costs. Additionally, the proposed solution is able to detect any current rise in cable screens which could endanger the cable screens, i.e., stray currents [20]. The proposed solutions are parts of global trends-similar topics are currently being researched by the International Council on Large Electric Systems-CIGRE working group [21] and newly created joint working group JWG D1/B1.75 "Strategies and tools for corrosion prevention for cable systems".

\section{Monitoring of Distribution System Network}

Despite $I_{r c s}$, the proposed solution requires additional input values-information about load currents of cable segments. The values can be obtained via a distributed monitoring system. Over the last decade, great progress has been observed in the field of distributed monitoring, and in consumer electronics-Internet of Thing (IOT) devices, industrial IOT, smart sensors 4.0, and advanced metering infrastructure (AMI) devices. IOT devices are developed for consumer applications, whereas industrial IOT and smart sensors 4.0 are developed for industrial applications. AMI meters were developed specifically for the needs of active distribution system networks. Different sensors are interconnected and form the sensor network. The literature presents many different architectures of sensor networks and many different communication protocols [22]. An example of a sensor network for power systems is the power system sensor network (PSSN) [23]. The PSSN integrates distributed monitoring and control systems. Sensors work coordinately, which allows for better observability of the network. The advantages of the PSSN are effective data access, processing, and network management. The system consists of a central control center and distributed sensors connected via dedicated communication channels and network infrastructure. Measurements are synchronized over GPS time synchronization. 
Sensors can be wireless (communication and energy supply), wired (communication and energy supply), or partially wireless-wired energy supply but equipped with modules which allow for wireless communication. Two types of sensors-wired AMI and wireless IOT devices-are compared in the context of a distribution network in order to present an overview regrading state of the art solutions and current issues connected with smart sensing technology. One of the biggest advantages of IOT devices is their plug and play capability and theoretically low cost. Unfortunately, commercial products-hardware enclosed in a housing with battery and algorithms—are still expensive- the price is in the range of a few hundred \$/sensor [24]. Despite the cost of the sensor, one needs to consider the installation cost, which is particularly high in distribution system networks because of installation issues-sophisticated equipment and work procedures which need to be adapted to work at height and on energized equipment. Moreover, the effectiveness of IOT devices is strongly limited by energy constraints-typical IOT devices have only small batteries and therefore cannot remain active over long periods of time, and therefore, wireless sensors are alternately switched on to perform actions and switched off to save energy [25]. It is possible to effectively measure some values in this way, i.e., temperature, since a time constant of the measurand is typically in the range of minutes or hours [26]. In some cases, continuous measurement is not needed, i.e., the low speed of degradation of the insulator surface allows the insulator leakage current to be measured periodically [27]. In order to increase the sampling rate of wireless sensors one needs to reduce the energy consumption. Typically, the reduction of energy consumption is achieved thanks to new, efficient communication protocols [28]. Moreover, it is possible to equip the sensor with an energy harvesting device, i.e., a magnetic field harvester, which is particularly interesting for power utilities $[29,30]$. Unfortunately, continuous operation and performing of advanced calculations, i.e., calculation of power quality parameters, is still impossible. The presented issues connected with IOT devices do not apply to AMI devices, which were developed for distribution system network monitoring. AMI devices are installed on the low voltage side of $\mathrm{MV} / \mathrm{LV}$ transformers, and therefore, an unlimited energy source is provided. AMI meters greatly improve the observability of distribution system network (DSN). AMI measurements are typically used for load analysis, load forecasting, and load management [31]. AMI is also used to monitor power quality, allows for detection of electricity theft, controls lights, or includes electric tariffs [32]. Many DSOs all over the world decided to install AMI meters in distribution system networks [33]. AMI is often presented in the literature in the context of efficient and secure data transfer and in the context of data compression [34-38]. Theoretically, AMI could be modified so new functions could be implemented; however, it is difficult to modify the software of AMI devices because meters would need to go through the testing process [39], and therefore, additional functionalities are achieved with the help of optional telemechanic devices [40] or smart sensors, i.e., smart tags [41]. It is also possible to interconnect different devices with the help of gateways or by upgrading conventional devices with new communication protocols, i.e., a phasor measurement unit (PMU) with an IOT interface [42], or include multiple interfaces and communication protocols in a single device [43]. A detailed description of sensing technologies and issues connected with sensing technology are presented in the CIGRE brochure (2021) [44] or the newly created CIGRE "WG D2.53 Technology and Applications of Internet of Things in Power Systems".

The presented considerations prompted the authors to utilize an existing AMI system, in particular load profiles for monitoring of the condition of the earthing system. The utilization of AMI data for earthing system monitoring is, to the best of the authors' knowledge, a world innovation. In order to monitor an earthing system in real-time, AMI data have to be sent to the central system, however, it is also possible to verify the earthing system condition periodically in offline mode-when AMI data are available. One should remember that the offline mode may not be optimal if AMI data are already compressed since the data would need to be recompressed for the purpose of analysis and compressed again. 


\section{Earthing System Current Sources}

Earthing screen current sources are presented in Figure 1. Earthing screen current sources marked with blue color are presented in the following paragraph, whereas other screen current sources are presented in previous papers [16-18]. Papers [16,17] present the earthing screen current sources under earth fault conditions-marked with red and green color in Figure 1. Paper [18] presents earthing screen current sources under load and no-load conditions-marked with yellow and grey color. This paper is a continuation of [18] and focuses on residual earthing screen current- $I_{r c s}$ under load conditions (yellow), which was initially omitted due to negligible small amplitudes. Further research, however, showed that even a very small current in the range of tens of $\mathrm{mA}$ can be used for the detection of earthing system mechanical damage, degradation, and cable line collapse. Because of the very small amplitudes of the current, it is mandatory to use precise current measurement instruments-preferably a Rogowski coil $[45,46]$. Attention is paid both to single segment cable lines and multi-segment cables lines.

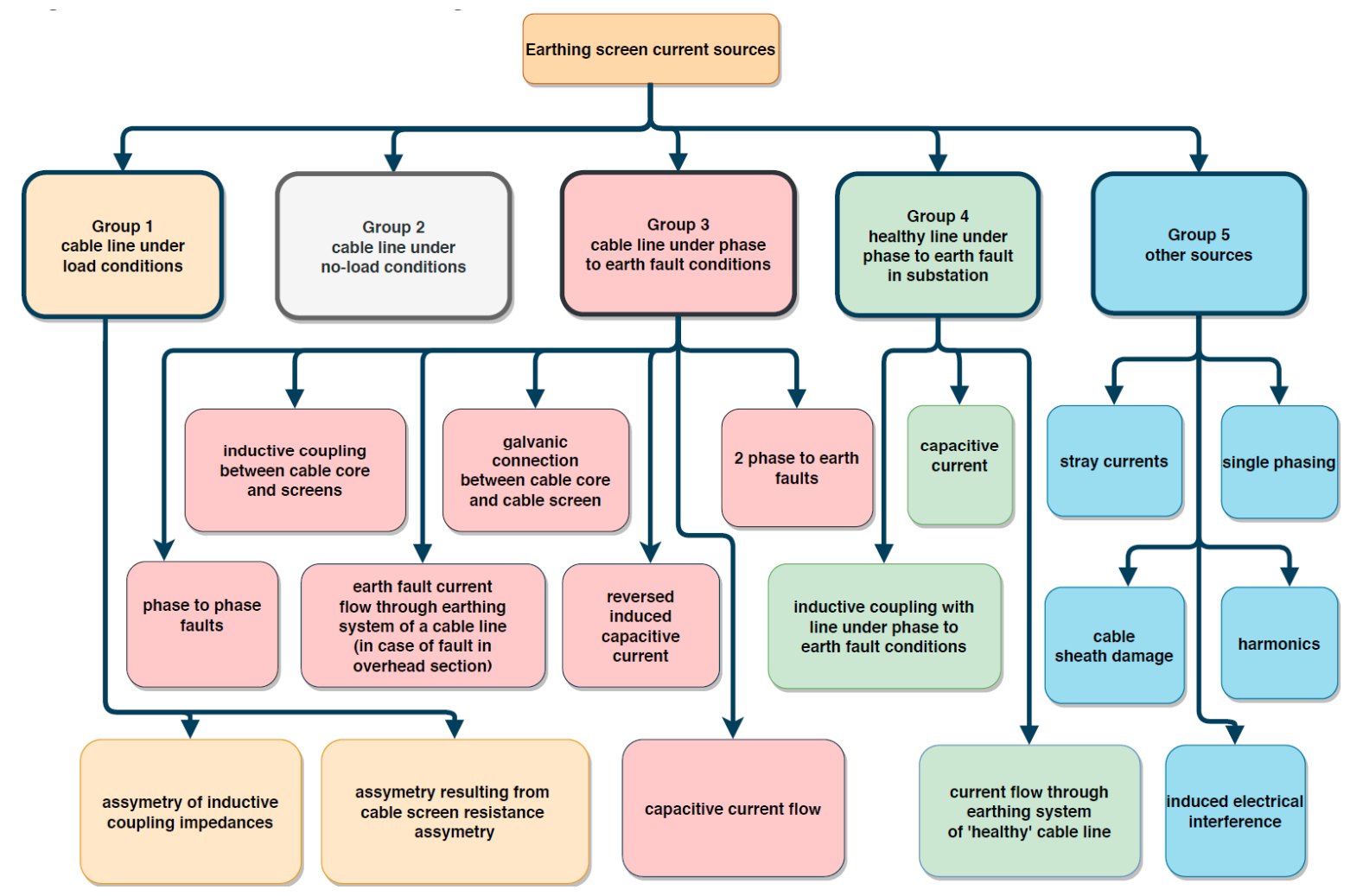

Figure 1. Earthing screen current sources.

The sources marked with grey, red, green, and blue colors are not used for the purpose of analysis; however, the sources have to be recognized in order to clean the data from disturbances, which have a negative impact on the accuracy of the results. Preparation of data is presented in Section 6.2. One of the sources of residual earthing screen current is the single phasing of the load [47]. Single phasing may be a result of a broken wire in an overhead line or improper operation of switching devices-damaged switch contact in one phase. It is however noted that under these circumstances, not only the rise of residual earthing screen current is observed, but also the rise of negative sequence in load current. Characteristic features allow single phasing to be detected and exclude the single phasing from the analysis of the earthing systems. Detection of single phasing is unfortunately limited to situations where a big part of the load goes under emergency single phasing conditions, which rarely occurs in real distribution feeders. In many cases, only a small share of a feeder load may go under single phasing conditions, and therefore, in some cases the detection of single phasing is almost impossible. 
In order to increase the effectiveness of single phasing detection, the use of measurements taken using AMI is recommended (taken at the LV side of MV/LV transformers) [47]. It is also possible to detect single phasing condition when cable line is de-energized - small resistance between phases indicates a proper connection, whereas out of scale resistance indicates that the connection is broken [48]. Single phasing is a dangerous operating condition since it can lead to damage of loads, particularly three phase motors [49], risk of electrocution due to, e.g., dangling wires of overhead lines, and potential load damages due to high voltage asymmetry.

Cable screen earthing current flow may also be a result of lightning impulse, which flows in the proximity of cable line and induces an impulse in the cable line. The induced lightning screen current impulse can be recognized thanks to the characteristic features of the waveform and very short duration in the range of $\mu \mathrm{s}$ [50] and therefore can be excluded from the earthing system analysis. It is also possible that in some cases additional screen earthing current sources will be active-stray current originating from traction or industrial loads [51]. Many stray current sources may be eliminated by filters since stray currents of traction loads are usually DC.

Harmonics distortions are always present in power systems; however, in some cases, harmonics distortion can be exceptionally high, which could result in abnormal $I_{r c s}$ flow. By observing the total harmonic distortion (THD) level of the $I_{r c s}$, it is possible to exclude highly distorted trends from the analysis. The $I_{r c s}$ may also be affected by induced disturbances, in such cases, the rise of $I_{r c s}$ is not proportional with the rise of cable core currents. Finally, the change of $I_{r c s}$ may be the result of cable sheath damage. According to [52], the damage can be detected with the help of waveform analysis.

\section{Simulation Results}

\subsection{Single-Segment Cable Line}

This paragraph presents the cable screen earthing current, factors affecting the $I_{r c s}$ current, and failure symptoms, which are the foundation of the solutions presented in Section 6. The simulations are carried out using PowerFactory software (2019 SP3). The developed model is presented in Figure 2. The model presents one section of a 110/15 kV substation and the most important components-simplified distribution feeders, 110/15 transformer, earthing transformer, and earthing impedance, as well as a model of analyzed cable (3x120/cable screen). The only component which is modified is the analyzed cable line, which is marked with a green frame. Other components are presented solely to show the structure of a section of the 110/15 substation in Poland. Other components (outside the green frame) do not have an impact on the simulation results. Switches marked with red are used to deenergize the cable core of segment cc1 and to supply the segment cc 2 via an ideal bypass connection. Simulations are performed in the quasi dynamic simulation module, which allows simulations to be performed in the long term-days, months, and years [53]. Loads are simulated based on real load profiles recorded by AMI meters installed in MV/LV transformer stations located in Poland. The load profiles presented in Figure 3 are modeled as relative loads. The nominal load is $1 \mathrm{MW}$, and for simplification, $\cos \varphi$ is constant and is equal to 0.9 .

According to the theory presented in previous papers [16,17], the amplitude of screen earthing current depends on:

- cable laying formation,

- parameters of cable line, in particular cable screen cross section,

- earthing system resistance,

- $\quad$ length of the cable line. 


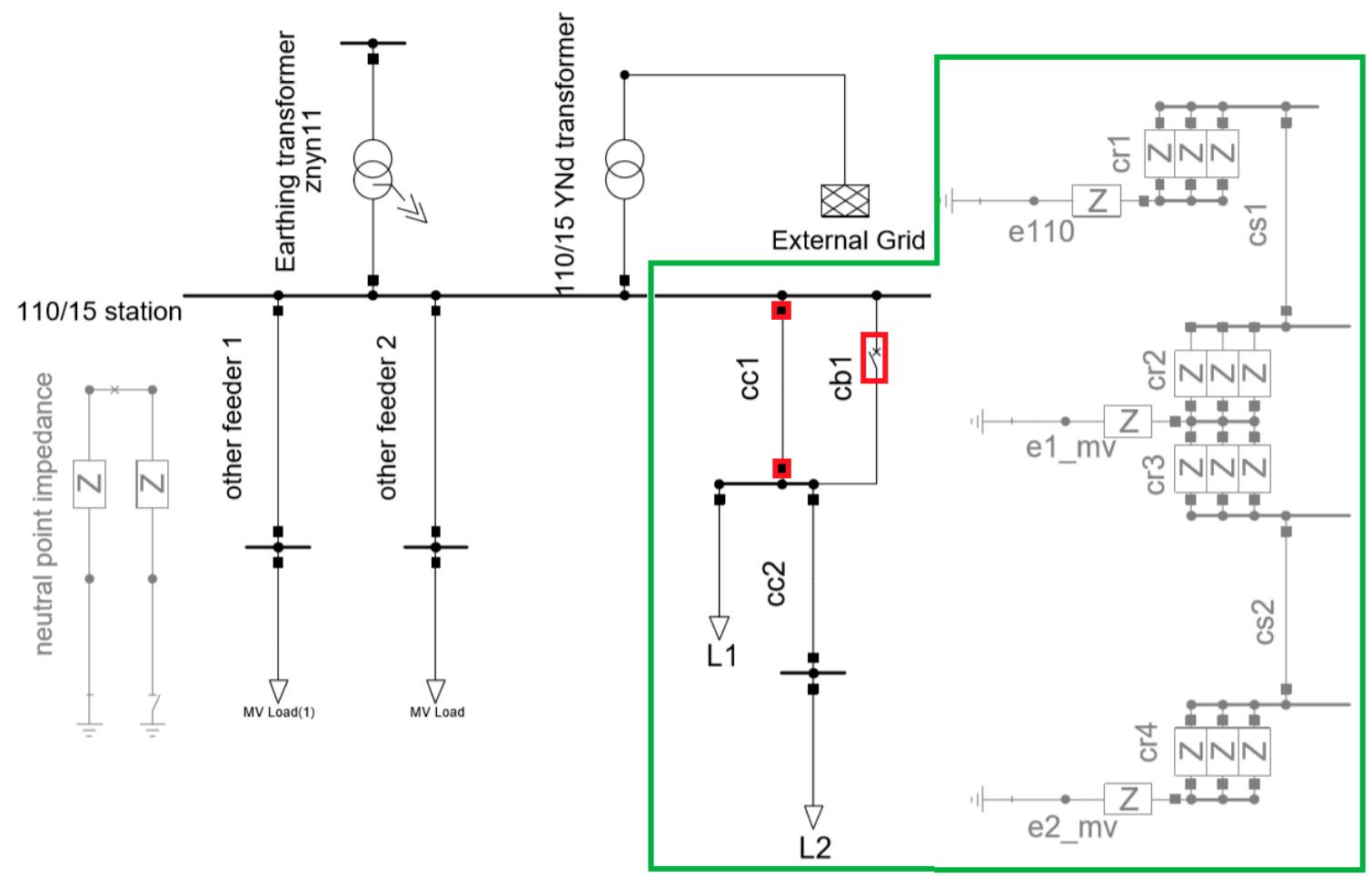

Figure 2. Power system model; cc—cable core, cs—cable screen, e110—substation earthing system resistance, and e1,2_mv-resulting resistance of MV transformer station.

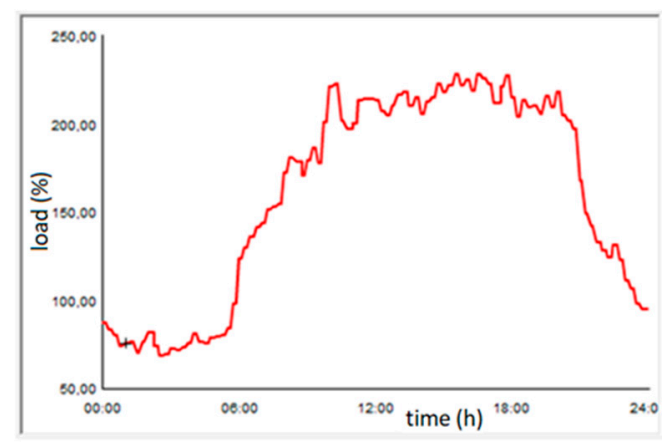

(a)

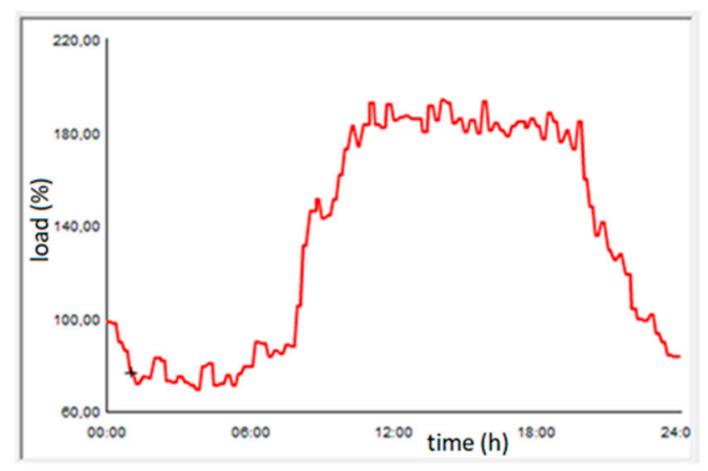

(b)

Figure 3. Analyzed load profiles of MV/LV station (left-type (a) load profile and right-type (b) load profile).

The longer the cable line is, the bigger the earthing screen current is. The phenomenon is explained by increasing source power, which is proportional to cable length, in relation to load power, which is a function of cable length and earthing system resistance. The $I_{r c s}$ is bigger in cables laid in the flat formation and smaller in cables laid in trefoil formation. The non-intuitional relation is explained by the fact that in trefoil formation, magnetic fields created by cables are neglecting each other almost completely, whereas in flat formation, the neglection effect is smaller due to bigger differences between coupling impedances between cables. Presently, trefoil formation is a standard solution in Poland; however, in practice, perfect trefoil formation does not exist, and therefore, an additional laying formation-degraded trefoil-is studied. Degraded trefoil is a flattened trefoil formation, presented in Figure $4 \mathrm{~b}$, which allows more realistic cases to be analyzed. Trefoil formation can worsen along the whole cable length or in particular places, i.e., because of cable collapse. 

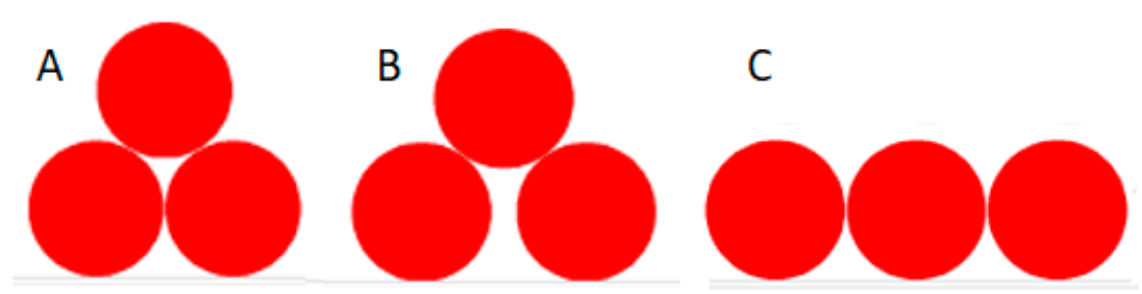

Figure 4. Analyzed cable laying formations. (a)-ideal trefoil formation, (b)-degraded trefoil formation, (c)-flattened trefoil formation.

Figure 5 presents the impact of cable length on residual screen earthing current- $I_{r c s}$. One can observe that $I_{r c s}$ increases with the increasing cable line length; however, for longer cable lines, one can observe that the current is almost the same $(1$ and $3 \mathrm{~km})$. Figure 6 presents the impact of earthing system resistance-for higher earthing resistances, the $I_{r c s}$ is reduced. One can also observe that increasing the resistance results in reduced extremes of $I_{r c s}$ and shifts the current toward the x-axis. Independently from the analyzed parameters (earthing system resistance and length), the shape of the load profile is reflected perfectly.
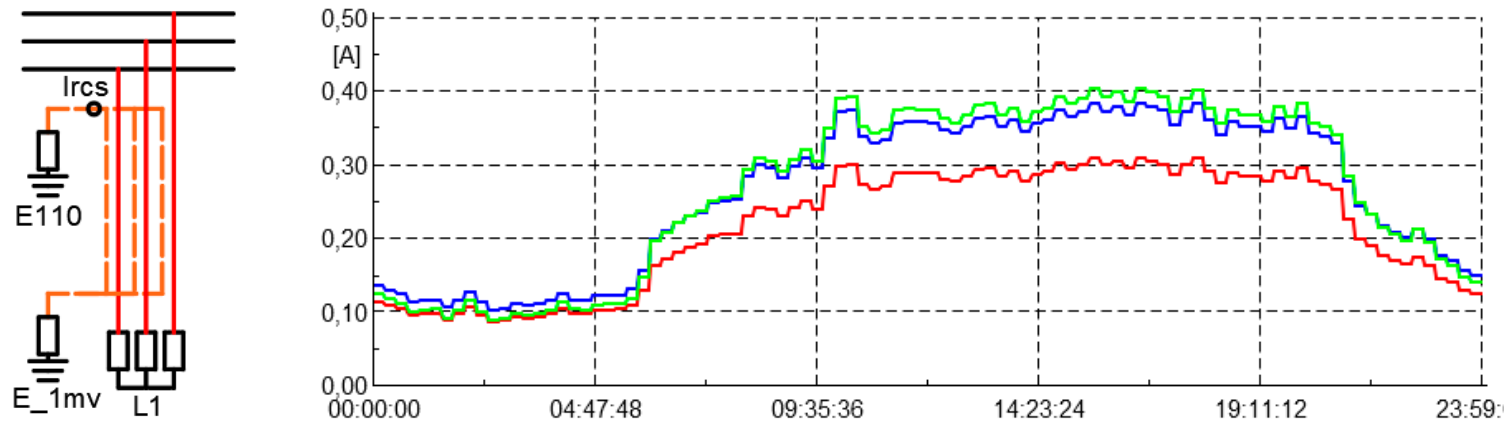

Figure 5. Impact of cable line lengths on $I_{r c s}$-residual earthing screen current. Starting from below $0.5 \mathrm{~km}$-red, $1 \mathrm{~km}$-blue, and $3 \mathrm{~km}$-green; $\mathrm{R}_{\mathrm{es}}=2 \Omega$. Feeder configuration is marked with colors red-energized cable—and orange—cable screen. Cable cross section: 3x150/25, earthing resistance-2 $\Omega$, load-relative load A (Figure 3) multiplied by $1 \mathrm{MVA}, \cos \varphi=0.9$.
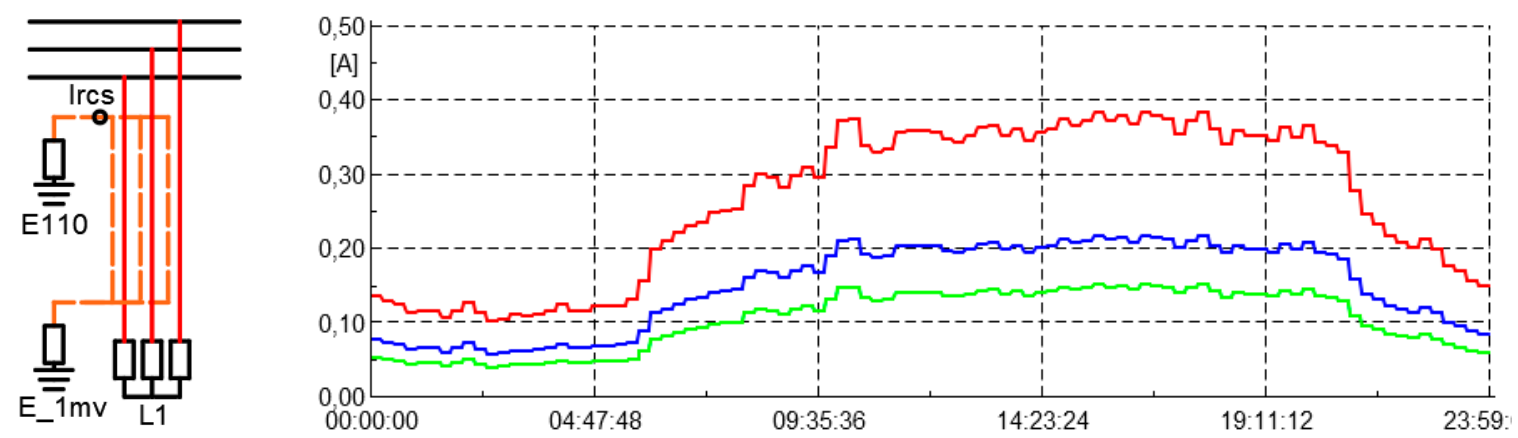

Figure 6. Impact of earthing system resistance on $I_{r c s}$ - residual earthing screen current; from above $2 \Omega$-red, $4 \Omega$-blue, and $6 \Omega$-green. Feeder configuration is marked with colors red-energized cable — and orange — cable screen. Cable cross section: 3x150/25, length: $1 \mathrm{~km}$, load—relative load A (Figure 3) multiplied by $1 \mathrm{MVA}, \cos \varphi=0.9$.

Figure 7 presents the impact of cable line flattening on the earthing screen current. One can observe that even relatively small deformation of trefoil formation (Figure $4 \mathrm{~b}$ ) leads to a high increase of $I_{r c s}$. Cable line collapse is considered a negative phenomenon because the flattening of the trefoil formation results in higher energy losses [54]. Unfortunately, in case of cable line collapse, there are no cost-effective repair methods. Practically the only solution to fix the cable formation would be to dig out the cable and to lay it from the beginning. Analysis of cable formation may however be an 
important indicator of work quality—cable laying—, and therefore, the analysis of cable formation would allow to find companies with a strong work culture.
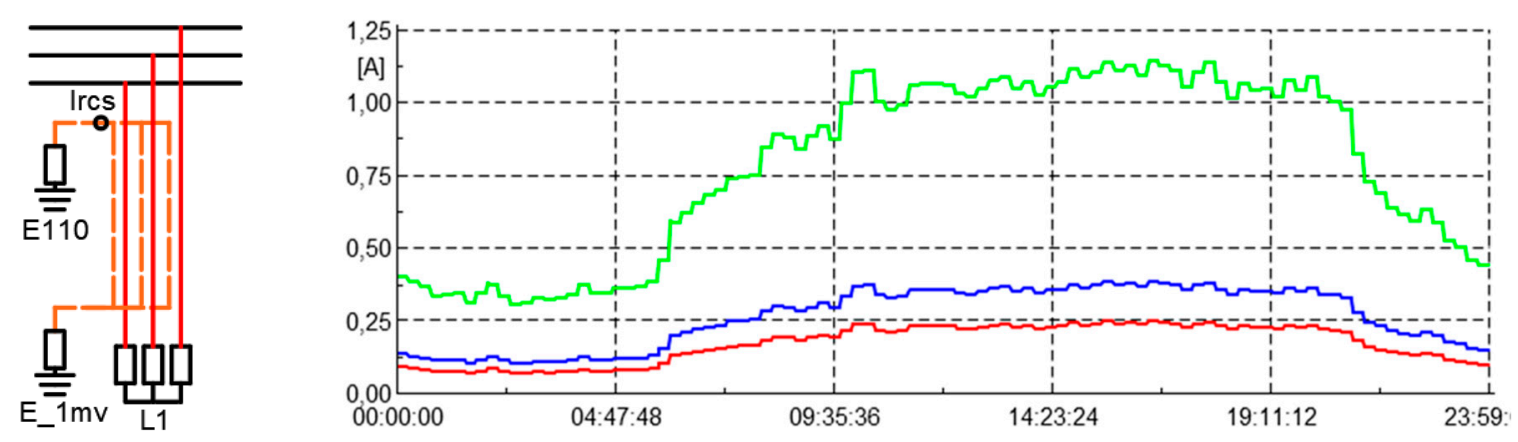

Figure 7. Impact of cable laying formation on $I_{r c s}$-residual cable screen earthing current; red—trefoil configuration, blue-flattened trefoil configuration, green-flat configuration. Feeder configuration is marked with colors red-energized cable-and orange—cable screen. Cable cross section: 3x150/25, length: $1 \mathrm{~km}$, earthing resistance-2 $\Omega$, load—relative load A (Figure 3) multiplied by $1 \mathrm{MVA}, \cos \varphi=0.9$.

The next reason for the rise of $I_{r c s}$ may be a failure of the cable screen connections. In that case, the current amplitude depends on the resistance of the cable screen connection and the location of the erroneous connection [18]. The rise of the $I_{r c s}$ may be very similar to the $I_{r c s}$ current rise resulting from the flattening of the cable formation. As a result, the classification of these types of failures may be difficult, solely based on current profiles. One has to however note that the processes of failure development for the two types of failures have a different time course. Collapsing of cable line is a long-term process, during which the earthing current rises slowly over the months, whereas in case of a cable screen connection failure, the rise of the $I_{r c s}$ may be observed instantaneously after the cable screen connection is damaged. Damage of the cable screen connection is connected with thermo-mechanical stresses under high current conditions; therefore, monitoring of thermomechanical stresses allows the cause of the earthing screen current rise to be determined.

\subsection{Multi-Segment Cable Line}

The scope of analysis is expanded to include multi-segment cable lines because the multi-segment lines are very common in distribution system networks. Moreover, the proposed solution is the most effective for the monitoring of multi-segment cable lines. Firstly, a cable line that consists of two (Figure 2) cable segments connected in series is analyzed. The switches marked with red color are configured in a way that only one segment of cable line is energized, whereas the cable screens are always connected. For both situations, the $I_{r c s}$ in the 110/15 substation is measured. The $I_{r c s}$ for scenario 1 is $I_{r c s 1}$ and the $I_{r c s}$ for scenario 2 is $I_{r c s 2}$. Finally, both segments of the cable feeder are energized and the resulting $I_{r c s}-I_{r c_{-} r} r$ is measured. For both scenarios, the loading currents $\mathrm{I}_{\mathrm{L} 1}$ and $\mathrm{I}_{\mathrm{L} 2}$ are exactly the same. Figure 8 presents cable screen earthing current for all scenarios. Additionally, the sum of $I_{r c s} 1$ and $I_{r c_{2} 2}-I_{r c_{S} S}-$ is presented. The $I_{r c_{-} r}$ is slightly different from the $I_{r c_{-} S}$. The differences are the result of interactions between $I_{r c s 1}$ and $I_{r c s 2}$, and these interactions are the topic of further research. Figure 9 presents the analogous situation; however, the loading currents $I_{L 1}$ and $I_{L 2}$ are different $-\mathrm{I}_{\mathrm{L} 1}$ is connected with load profile A and $\mathrm{I}_{\mathrm{L} 2}$ with load profile $\mathrm{B}$.

Figure 10 presents the results of the degradation of the first MV earthing system, whereas Figure 11 presents result of the degradation of the second earthing system (e2mv). As can be observed, degradation of the first earthing system (e1mv) has a bigger impact on $I_{r c s}$ since it influences the current loops in two cable segments, whereas degradation of the second (last of the feeder) earthing resistance influences only one cable segment. The presented results prove that it is possible to indicate the location of damaged or degraded earthing systems. Failure of the cable screen connection has a major impact on only one cable line segment. 


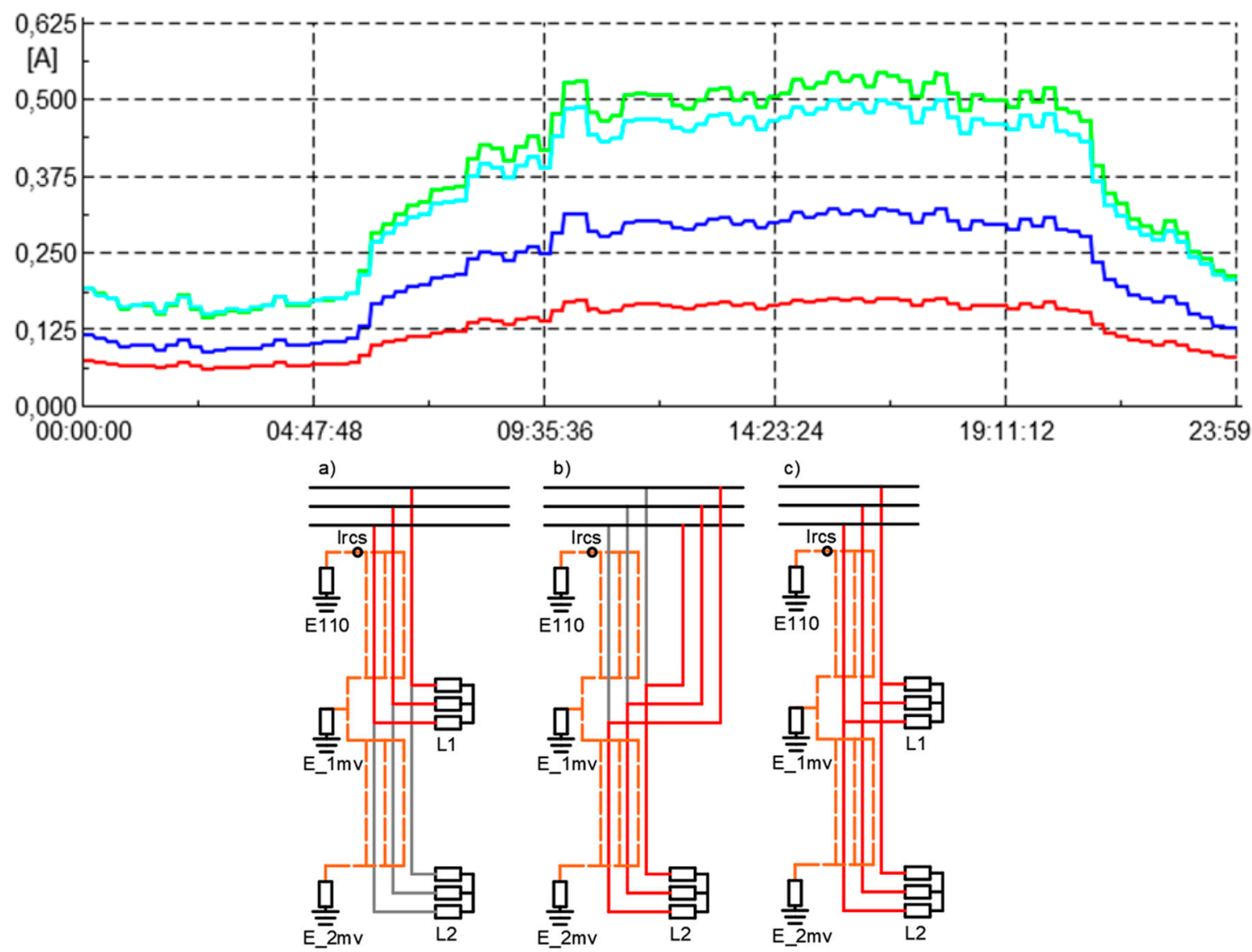

Figure 8. Residual cable screen earthing currents $-I_{r c s}$ - of cable segments loaded with load A; red—segment cc2 (configuration b); blue-segment cc1 (configuration a); light blue—sum of $I_{\text {rcs }}$ for configurations $\mathrm{a}$ and $\mathrm{b}$; green- $\mathrm{cc} 1$ and $\mathrm{cc} 2$. Feeder configuration is marked with the following colors: red—energized cable, grey-deenergized cable, and orange—cable screen. Cable cross section: 3x150/25, length of segments: $1 \mathrm{~km}$, earthing resistances $-2 \Omega$, load-relative load A (Figure 3) multiplied by $1 \mathrm{MVA}, \cos \varphi=0.9$.

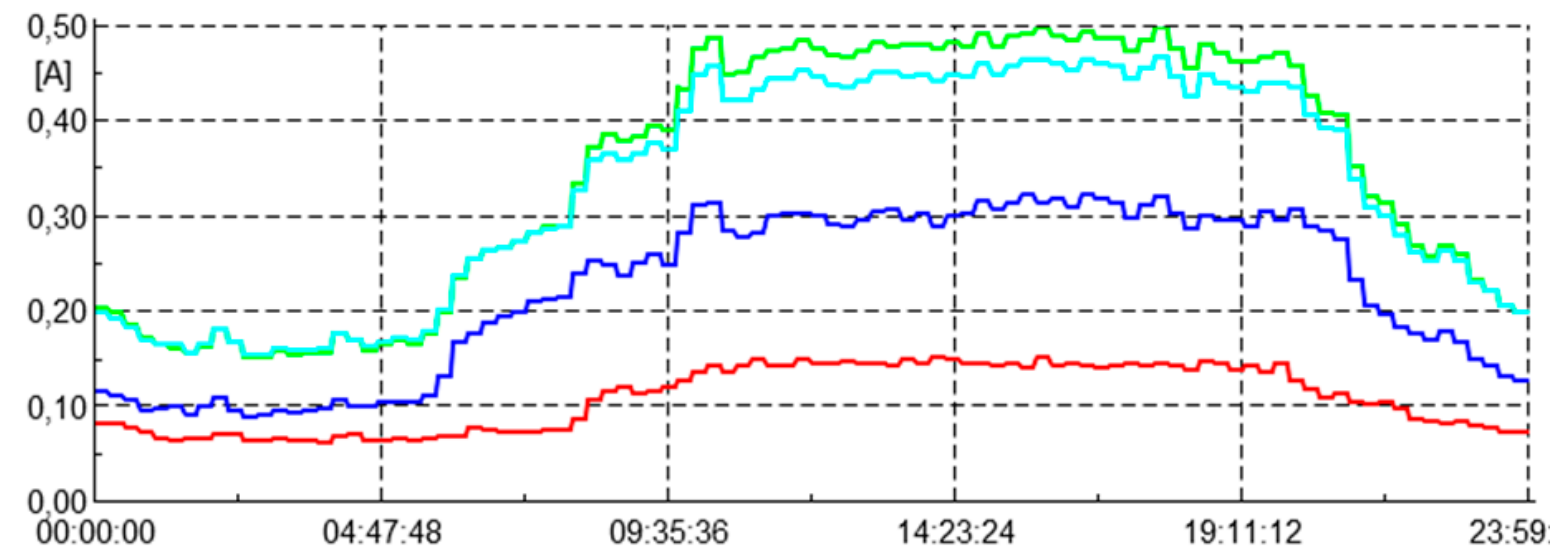

Figure 9. Cont. 
a)

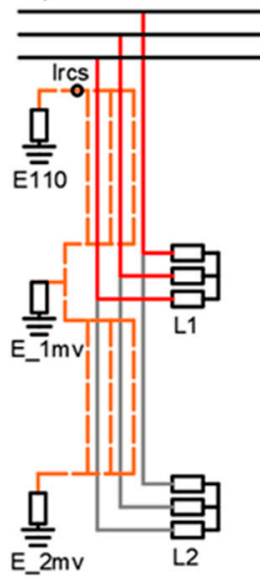

b)

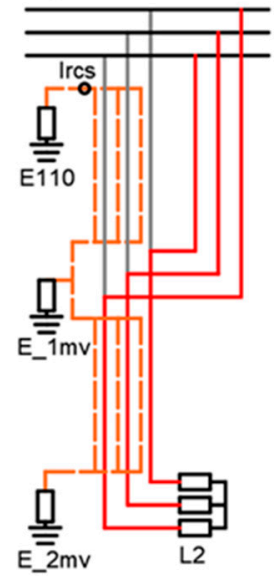

c)

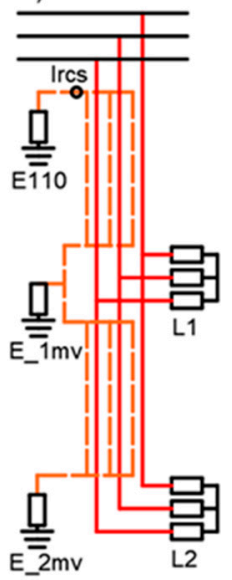

Figure 9. Residual currents of cable segments $-I_{r c s}$-in case of loads load A in section cc1 and B in section cc2; red-segment cc2 (configuration b); blue-segment cc1 (configuration a); light blue-sum of $I_{r c s}$ for configurations a and b; green—cc1 and cc2. Feeder configuration is marked with the following colors: red—energized cable, grey—deenergized cable, and orange—cable screen; a) $-1^{\text {st }}$ cable segment is energized, $b)-2^{\text {nd }}$ cable segment is energized, c) both cable segments are energized. Cable cross section: 3x150/25, length of segments: $1 \mathrm{~km}$, earthing resistances $-2 \Omega$, load-relative load of the first cable segment (cc1) - A (Figure 3) multiplied by $1 \mathrm{MVA}, \cos \varphi=0.9$, relative load of the second cable segment (cc2)-B (Figure 3) multiplied by 1 MVA, $\cos \varphi=0.9$.
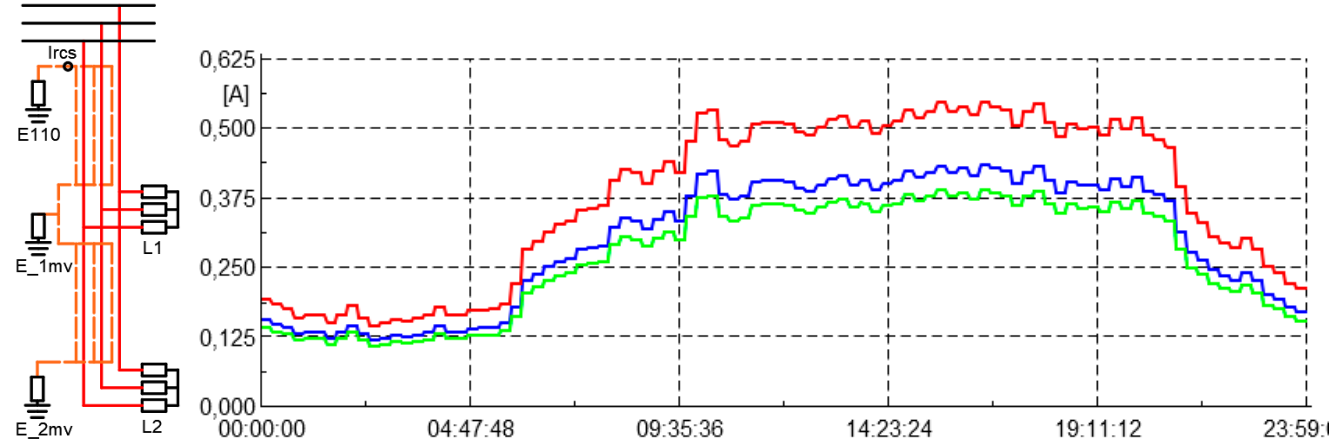

Figure 10. Impact of $R_{\mathrm{e} 1 \mathrm{MV}}$ on cable screen earthing current (from above 2, 4 and $6 \Omega$; cable length $1 \mathrm{~km}$ ). Feeder configuration is marked with colors red-energized cable - and orange-cable screen. Cable cross section: $3 \times 150 / 25$, length of segments: $1 \mathrm{~km}$, earthing resistances $-2 \Omega$, load-relative load A (Figure 3) multiplied by 1 MVA, $\cos \varphi=0.9$.
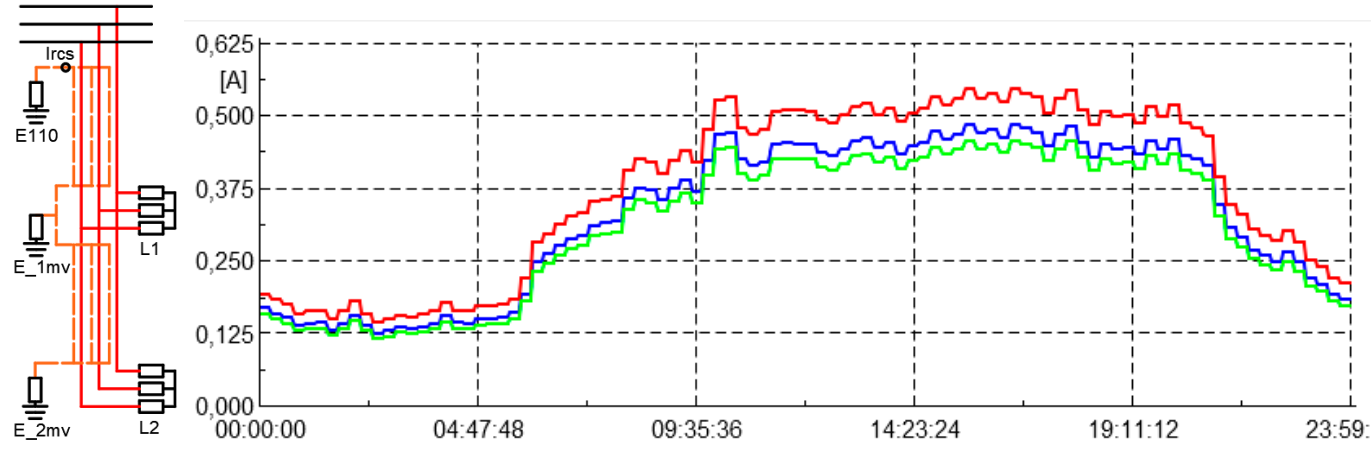

Figure 11. Impact of $\mathrm{R}_{\mathrm{e} 2 \mathrm{MV}}$ on $I_{\text {rcs }}$ (from above 2, 4 and $6 \Omega$; cable length $1 \mathrm{~km}$ ). Feeder configuration is marked with colors red-energized cable-and orange-cable screen. Cable cross section: 3x150/25, length of segments: $1 \mathrm{~km}$, earthing resistances $-2 \Omega$, load-relative load A (Figure 3) multiplied by 1 MVA, $\cos \varphi=0.9$. 
Figure 12 presents the results of the break of earthing system continuity-Re1mv-green, Remv2-blue. The red curve presents the $I_{r c s}$ current under normal operating conditions-earthing systems are connected properly. The presented type of failure is also characterized by a reduced earthing screen current amplitude; however, in this case, failure occurs immediately, i.e., when the earthing system is broken. The presented results allow the conclusion to be made that knowledge about the load profiles of cable segments allows the segment of cable line which is responsible for the abnormal flow of $I_{\text {rcs }}$ to be indicated.
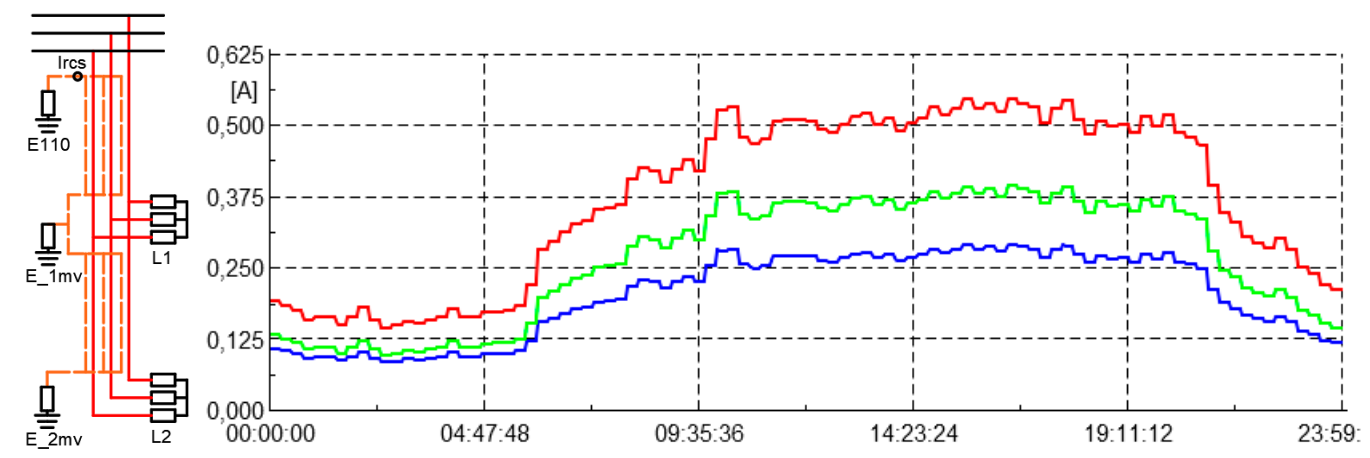

Figure 12. $I_{r c s}$ in case of the break of earthing system continuity: Re1mv-green, Remv2-blue; red-reference curve-no failure. Feeder configuration is marked with colors red-energized cable - and orange - cable screen. Cable cross section: 3x150/25, length of segments: $1 \mathrm{~km}$, earthing resistances-2 $\Omega$, load-relative load A (Figure 3) multiplied by 1 MVA, $\cos \varphi=0.9$.

The principles of cable screen current flow in multi-segment cable lines are similar to single segment cable lines; however, additionally, one needs to consider the fact that the amplitude of $I_{\text {rcs }}$ forced by the cable segment depends on the impedance of the cable screens and earthing systems laid before the segment. Segments which are laid before the analyzed cable segment create parallel paths for current flow. In general, when the number of earthing systems increases and the length of the cable segments before the considered segment increases, the share of residual current of the segment which flows through the earthing system of the 110/SN substation is reduced. It is difficult to specify the maximum length of cable feeder or number of sections which allow the whole cable feeder to be monitored. The amplitudes of earthing screen currents also depend on the main source of the currents-the load currents of different cable segments. In general, one can say that the bigger the load current is, the bigger the screen earthing current is. It is recommended that the screen earthing current is analyzed under high loading conditions since the earthing screen current is larger. Moreover, the bigger the differences between load profiles are, the easier it is to indicate the cable section responsible for the abnormal earthing screen current flow. In real cable lines, the variability of load profiles increases with the rise of distance from the substation, which increases the sensitivity of the proposed solution. After taking all the factors into account, one can conclude that it is not possible to monitor the whole of the long complex cable feeder with just one point measurement. Cable segments located close to the substation are monitored. Those segments are the most important because a failure of those segments would affect the biggest number of consumers. Cable segments in the depths of the network can be monitored with additional earthing screen current sensors.

\subsection{Thermo-Mechanical Stresses}

In order to calculate the currents which generate thermo-mechanical stresses, phase to phase faults are simulated at the end of the cable line. Simulations are carried in the Root-Mean Square (RMS) module of PF software. More detailed transient analysis can be made using the Electromagnetic Transients (EMT) module of PowerFactory or different software [55]. Figure 13 presents cable screen currents under two phase to phase fault conditions, and Figure 14 presents cable screen currents under three phase to phase fault conditions. The cable core cross section is $120 \mathrm{~mm}^{2}$, whereas the cable screen 
cross sections are modified and presented in the legend of Figures 13 and 14. The relative fault location is always the same-at the end of the cable. Faults at the end of the cable line can also be interpreted as faults at the beginning of the overhead line of mixed cable overhead feeders. Phase to phase faults along cable lines rarely occur. It is more likely that the fault will occur in the overhead section of a mixed feeder. The probability of a fault in the line is given in faults per year and per kilometer of the line; therefore, the longer the line the bigger is the risk of phase to phase faults. Based on the results presented in Figures 13 and 14, one can say that with increasing cable line length, currents flowing through the cable screens under two phase and three phase fault conditions are reduced. This tendency can be explained by the rise of cable impedance to fault location. It has to be underlined that the ratio of cable core and cable screen currents remains the same, regardless of the fault location. The presented results allow the practical conclusion to be drawn-the shorter the cable line of cable overhead feeder, the higher the cable screen current amplitude is, and therefore, the thermo-mechanical stresses are greater. Additionally, one can say that the longer the overhead line is, the bigger the quantity of thermo-mechanical stresses that can be expected. Thermo-mechanical stresses damage the cable screen connections-in particular in cable joints and cable heads. The bigger the cable screen cross section, the higher the amplitudes of the cable screen currents are, and therefore, paradoxically, cables with smaller cable screen cross sections are better protected against thermo-mechanical stresses.

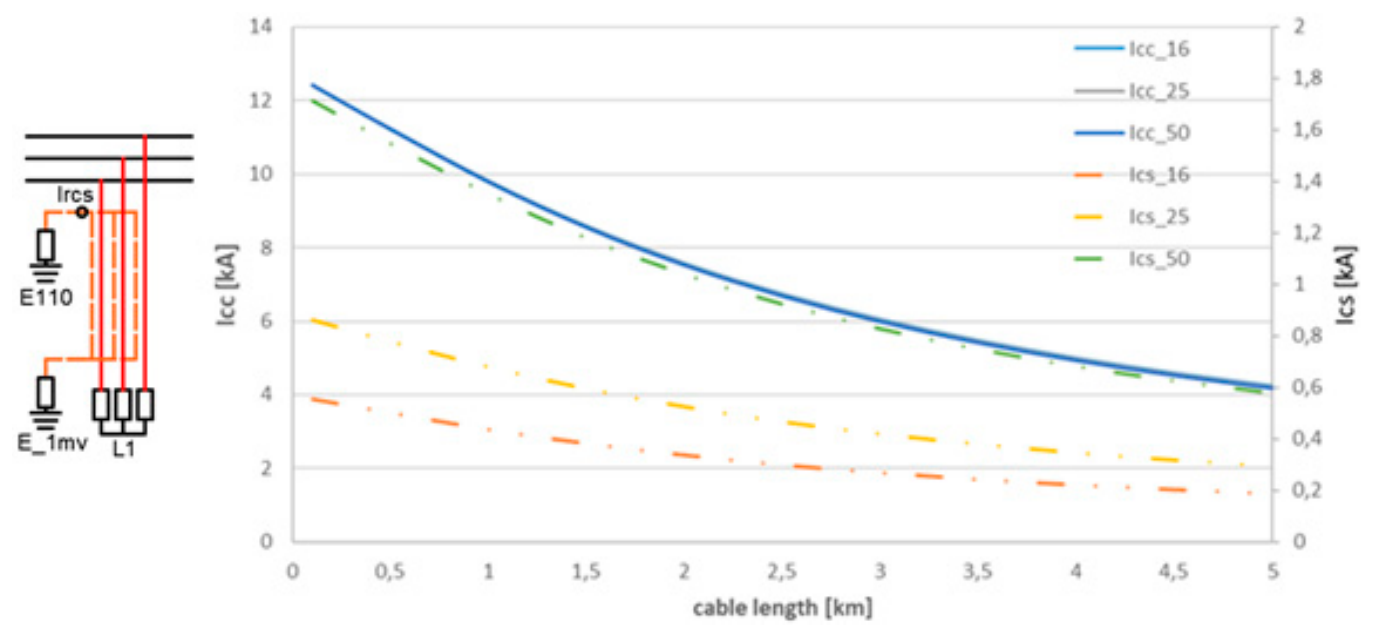

Figure 13. Current induced in cable screens under $2 \mathrm{f}$ fault conditions at the end of cable line $\left(I_{c c}-\right.$ cable core current; $I_{\mathcal{C S}}$-cable screen current). Configurations are marked with the color red-energized cable-and orange-cable screen.
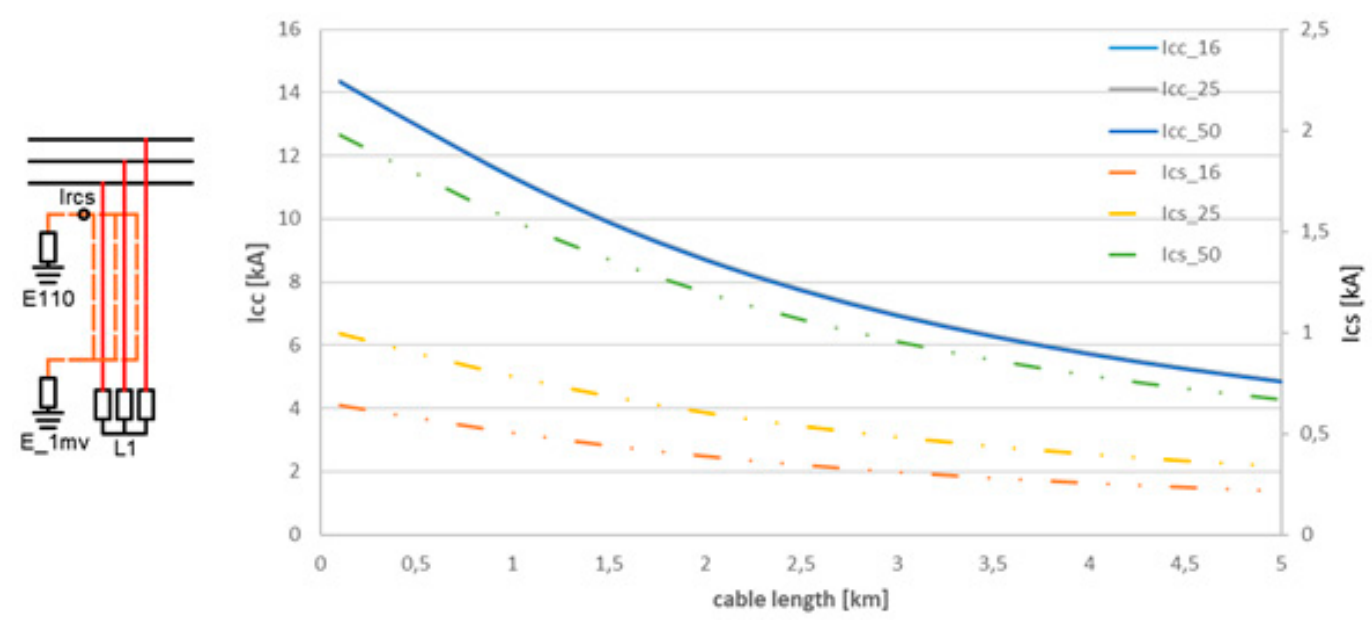

Figure 14. Current induced in cable screens under $3 \mathrm{f}$ fault conditions at the end of cable line ( $I_{\mathcal{C}}$-cable core current; $I_{\mathcal{C S}}$-cable screen current). Configurations are marked with color red-energized cable-and orange-cable screen. 


\section{Measurement Results}

In order to confirm the results of theoretical considerations presented in Section 4 and to prove that despite many potential disturbances, i.e., earth faults, it is possible to monitor the $I_{r c s}$, the measurements in real cable line are taken. Measurements are taken in cable line which is a part of cable overhead feeder. The cable line is $0,78 \mathrm{~km}$ long (one segment) and the line type is $3 x Y H A K X S 120 / 5012 / 20 \mathrm{kV}$. Figure 15 presents averaged true RMS current trends recorded in cable cores and screens over a 10-day period. Measurements in cable cores are taken on the secondary side of current transformers, whereas measurements of currents in cable screens are taken directly at cable screens. Rogowski coils are installed in a way that the cable screens are in the middle of the Rogowski coil measuring windows to ensure the lowest possible measuring errors. As can be observed in Figure 15, the phase currents measured in cable cores overlap each other, and therefore, one can conclude that the load is almost perfectly symmetrical. Variation of load is not typical for this distribution feeder because of the renewable energy source-wind turbines with relatively high power-in comparison with the loads of the feeder. Nevertheless, as expected, cable screen currents reflect the trends of cable core currents very well, at the same time, however, the cable screen current amplitudes differ significantly. The cable screen currents asymmetry is typical for cables laid in flat formation [56] and therefore one can conclude that cable is laid in flat formation. The analyzed cable line is built according to old standards. Currently, single core cable lines in MV networks are laid in trefoil formation in order to reduce cable screen losses [57].

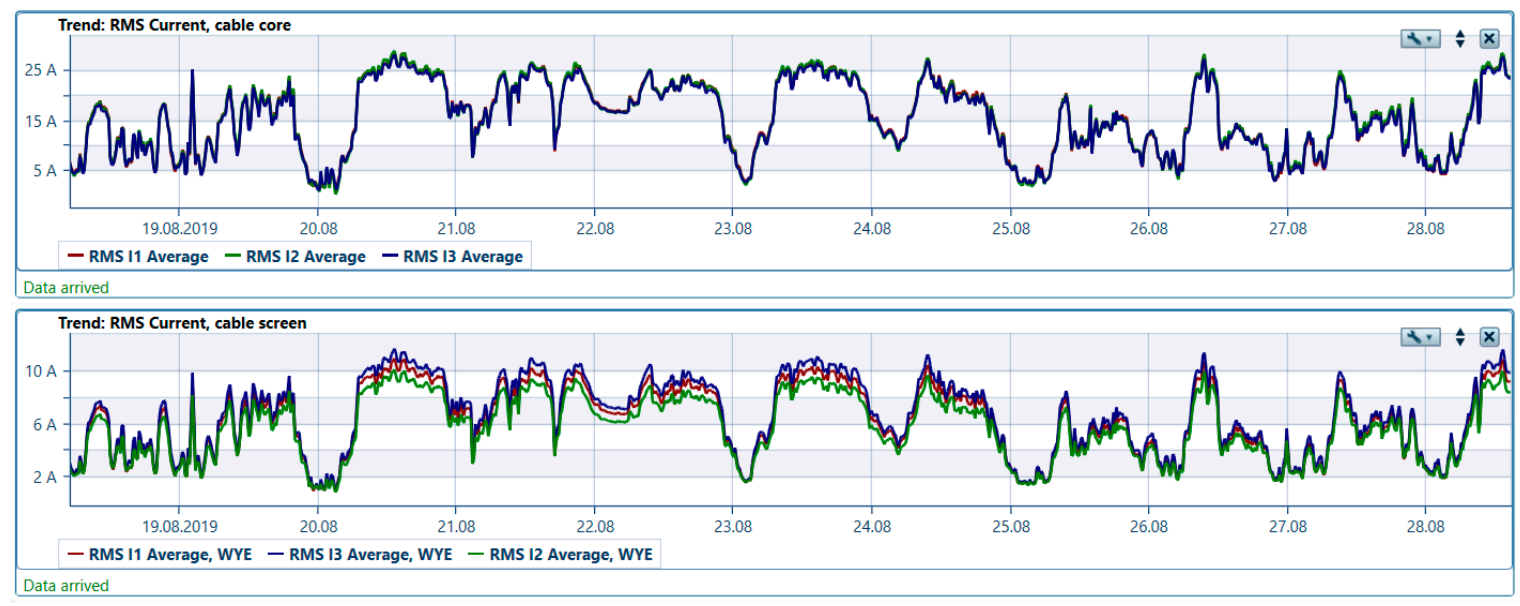

Figure 15. Currents in cable cores and screens; (Rogowski coils measuring range set to 30A [58]).

Figures 15 and 16 are scaled to present average 15 min values. Figure 15 presents the load current as phase values. Load currents can also be expressed as a single value-positive sequence current, which is obtained according to theory of symmetrical components, or as total load which is a sum of phase loads. Expression of load as a single value greatly simplifies the correlation analysis. Positive sequence load current is presented at the top of Figure 16. Below the positive sequence load current, one can observe the zero-sequence screen current, $I_{0 c s}$, and at the bottom, the cable screen earthing current $I_{r c s}$. The $I_{r c s}$ is measured at the point of connection of the cable screens and earthing system of the $110 / 15$ substation. The $I_{0 c s}$ is obtained according to symmetrical components theory by summing the cable screen phase currents. Both currents express current flowing through the loop created by the cable screens and the earth. According to this theory, both currents are equivalent; therefore, for the presented analysis, only $I_{r c s}$ is required. Both values are presented to confirm that the measurement results are reliable. Slight differences between $I_{r c s}$ and $I_{0 c s}$ may be explained by the limitation of the measuring equipment- the measuring errors of three cable screen currents add up when the sum of currents $\left(I_{0 c s}\right)$ is calculated. Moreover, the beginning of the Rogowski coil measuring range is $0.1 \mathrm{~A}$, and therefore, smaller current amplitudes are burdened with a greater error. The $100 \mathrm{~mA}$ value is marked in 
Figure 16 with a black line, horizontal to the $\mathrm{x}$ axis. Nevertheless, despite the small differences, one can observe that two measurements give very similar results, which confirms that the measurements were taken properly. Analysis of Figure 16 allows us to conclude that the correlation between the load current expressed as positive sequence current $-I_{p c c}$-and the $I_{r c s}$ is very high. It is important to note that both measured currents- $I_{p c c}$ and $I_{r c s}$-are true RMS currents, which include harmonic distortions, etc. Theoretically, it is possible to extract the fundamental current components; however, AMI meters typically record the total load, which includes harmonic distortion; therefore, utilization of the true RMS value, even though it could be slightly less accurate, is definitely easier to implement. For the purpose of diagnostics, it is convenient to present the $I_{r c s}$ as a function of $I_{p c c}$ on the $\mathrm{x}-\mathrm{y}$ Cartesian plane (Figure 17). As can be observed, $I_{r c s}$ is clearly a function of load current; however, real life measurements are not as perfectly repeatable as the simulation software, and therefore, one needs to consider the $I_{r c s}$ and $I_{p c c}$ relation as a measuring band limited by two parallel lines [59]. The majority of measuring points are within the band. Some points outside the band can be removed from further analysis because the points are connected with transients, strongly distorted waveforms conditions, etc. Moreover, one can observe that a number of points outside the band are bigger when $I_{r c s}$ is below $100 \mathrm{~mA}$ (outside the measuring range), which is marked by a green line (Figure 17), horizontal to the $\mathrm{x}$ plane. The data presented on the Cartesian plane ( $x-y)$ are typically used as input for machine learning systems [60].
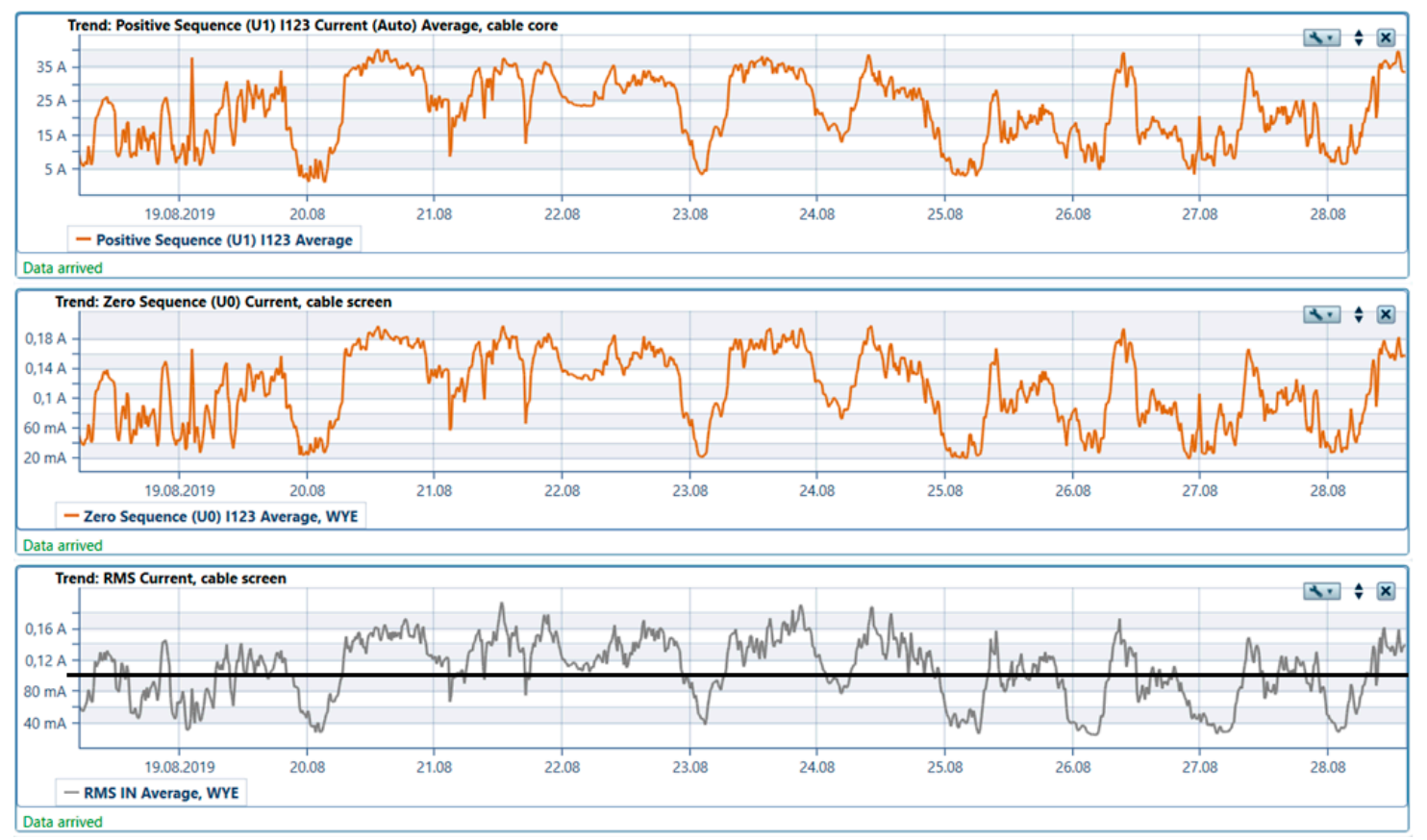

Figure 16. Criterion values used in the presented algorithm: positive sequence load current (first from above), digitally calculated zero-sequence current (in the middle) and residual earthing screen current- $I_{\text {rcs }}$ (at the bottom).

Measurement results presented in Figures 15-17 were taken under mild weather conditions (only short, $<1 \mathrm{~h}$ periods of intermittent rain). Unfortunately, the authors suspect that in some cases, the correlation between the load current and $I_{r c s}$ may not be so straightforward. It is possible that the earthing system resistance will vary significantly because of ambient conditions, i.e., rainfall or snow melting. In the case of rainfall, it is relatively simple to correlate the earthing resistance variance with the rainfall since the effects of rain can be observed shortly after rainfall. The effects of snow melting may be recognized using information about snow and temperature-if after snowfall the ambient temperature rise above $0{ }^{\circ} \mathrm{C}$, the resistance of the earthing system will be reduced, and therefore, the earthing current will increase. One has to note however that the earthing system resistance 
variations may be observed mainly in the simple earthing system, i.e., at the end of cable line segment or cable junction boxes. Variability of resistance in urban earthing systems is greatly reduced because an earthing system is a complex structure, which is built out of an MV/LV station earthing system and an earthing system of Protective Earth and Neutral (PEN) wires connected with the station [61]. Earthing systems of PEN wires are often connected with the foundation earthing of buildings that ensure stable resistance [62]. Moreover, the resistance of earthing systems in urban areas is often not sensitive to ambient conditions because of mechanical barriers, i.e., asphalt or concrete covering the earthing systems. Additionally, drainage water systems allow the soil moisture to be kept stable.

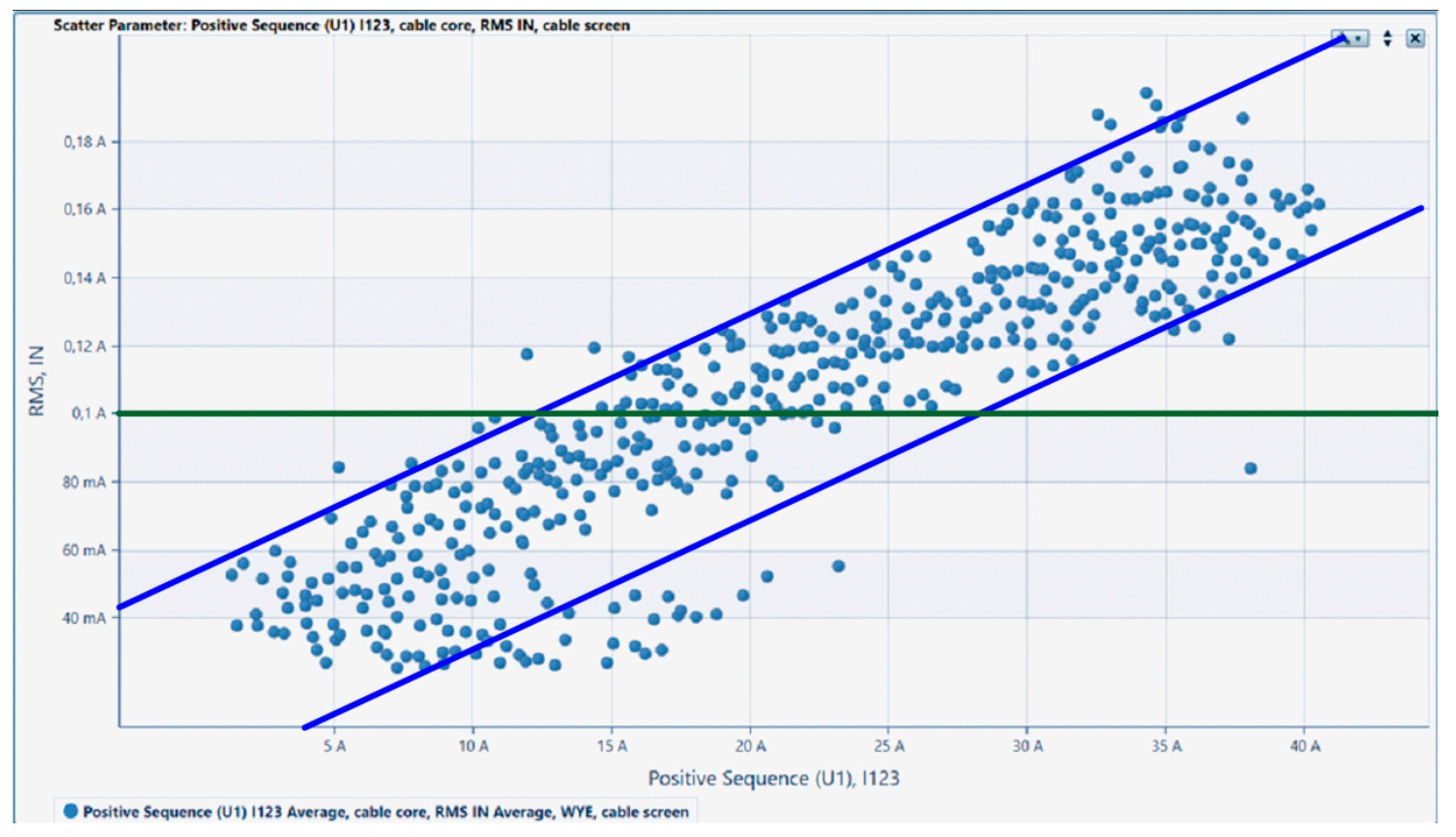

Figure 17. $I_{r c s}$ as a function of $I_{p c c}$ (residual earthing screen current as a function of positive sequence load current).

\section{Developed Solutions}

\subsection{General Structure}

The general structure of the proposed system is presented in Figure 18. The main elements of the system are protection relays, which measure the cable screen earthing current. The server is a central unit that performs the functions presented in the paragraphs below. Computer and mobile devices allow for manual data access, analysis, and visualization. Other components provide information for data analysis. Components marked with black font are necessary for the operation of the system: weather data, models of the cable feeder (equipment and maintenance database), and current configuration of power system (SCADA). Components marked with blue font are optional and can extend the functionalities of the proposed system-A GIS (geographical information system) could store the location of the feeder and location of failures, a failure database could be used to analyze the correlation between thermo-mechanical stresses and failures, whereas external sensors could provide data from additional current measurements from the depths of the network.

The proposed solution can be presented as two general algorithms, presented in Figure 19—one for real time monitoring and data acquisition and the second for data preparation and data analysis. Both algorithms are run in parallel. Real-time monitoring is performed using protection relays (IED), which measure the cable screen earthing current, whereas data preparation and analysis are performed by a centralized application-the server. Thanks to the real-time monitoring, it is possible to detect a few failure types immediately-loss of connection with the earth and damage of the cable screen 
connections. Data preparation is the process of data acquisition and data cleaning, which is described in the section below. Processed data are used for data analysis in order to detect degradation of the cable line formation, degradation of the earthing system or degradation of the cable screen connection due to cumulative ageing. Detailed algorithms are presented below.

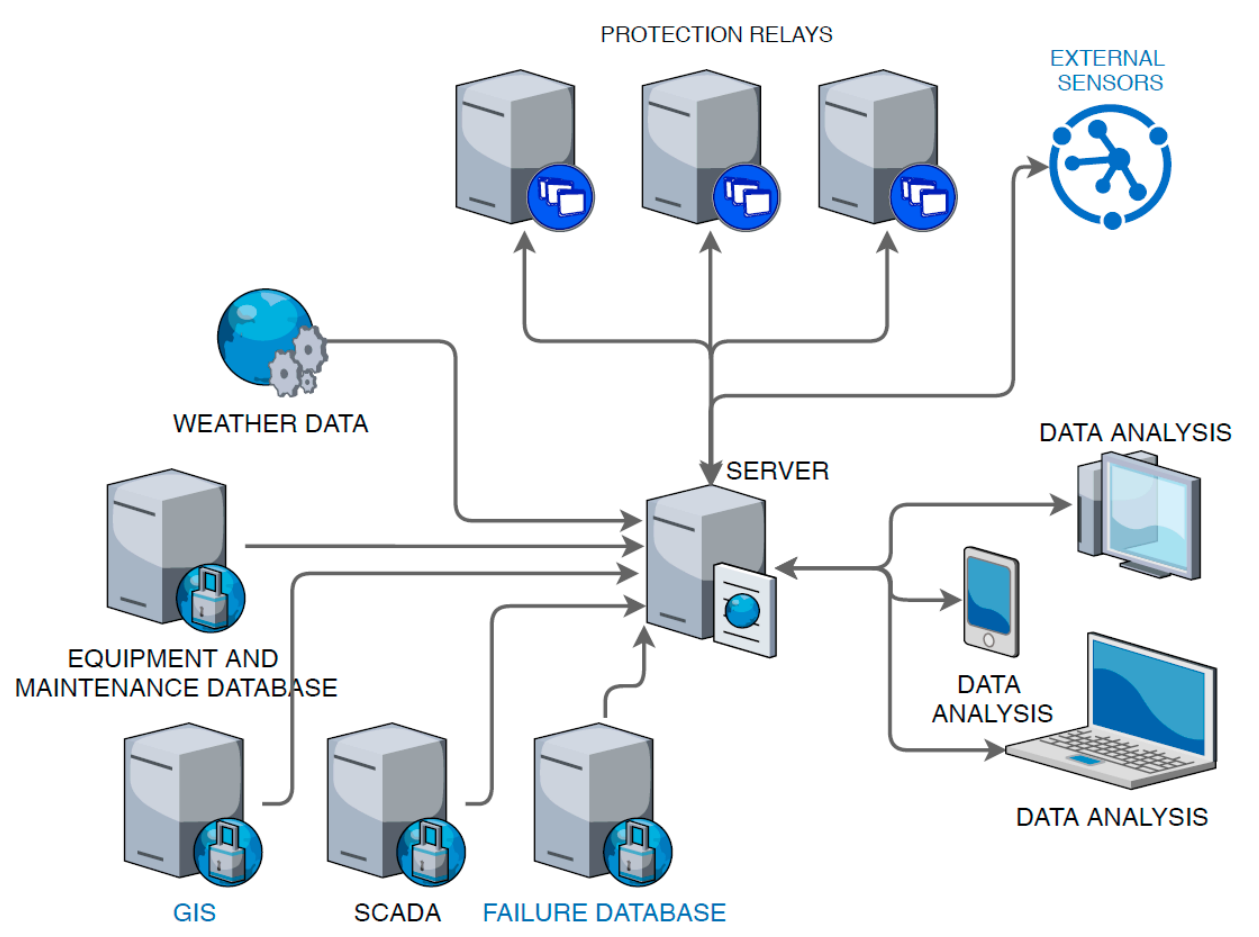

Figure 18. Structure of the proposed system; elements marked with black font are obligatory and elements marked with blue font are optional.
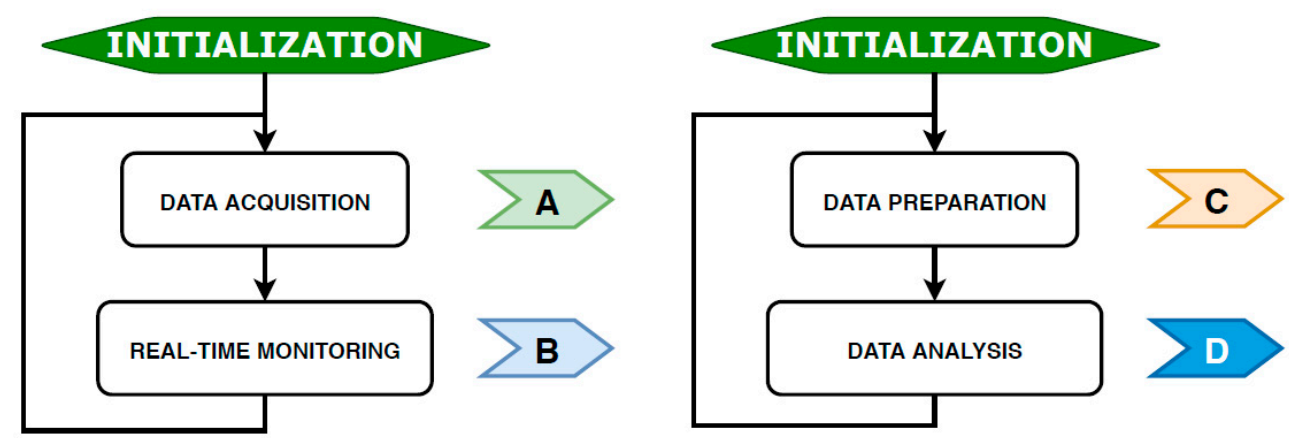

Figure 19. General flowchart of the proposed solution; letters indicate detailed algorithms presented below.

\subsection{Data Processing and Analysis}

The detailed flowchart of data processing and analysis is presented in Figure 20. The steps are described below in ascending order.

In order to initialize data preparation and analysis, one needs to obtain information about the cable feeder and network structure. The information can be obtained from the distribution system operator (DSO) line and equipment databases. One has to underline that the network structure should be saved in a standardized Common Information Model (CIM) format [63].

After the initialization process, the data are processed. At the beginning, the data are cleaned, i.e., averaging periods which contain fault incidents are removed from the data set. Faults are recognized thanks to information from the protection relay register [64]. Data averaging periods affected by earth faults and other disturbances, i.e., stray current flow incidents, are excluded from the analysis. 

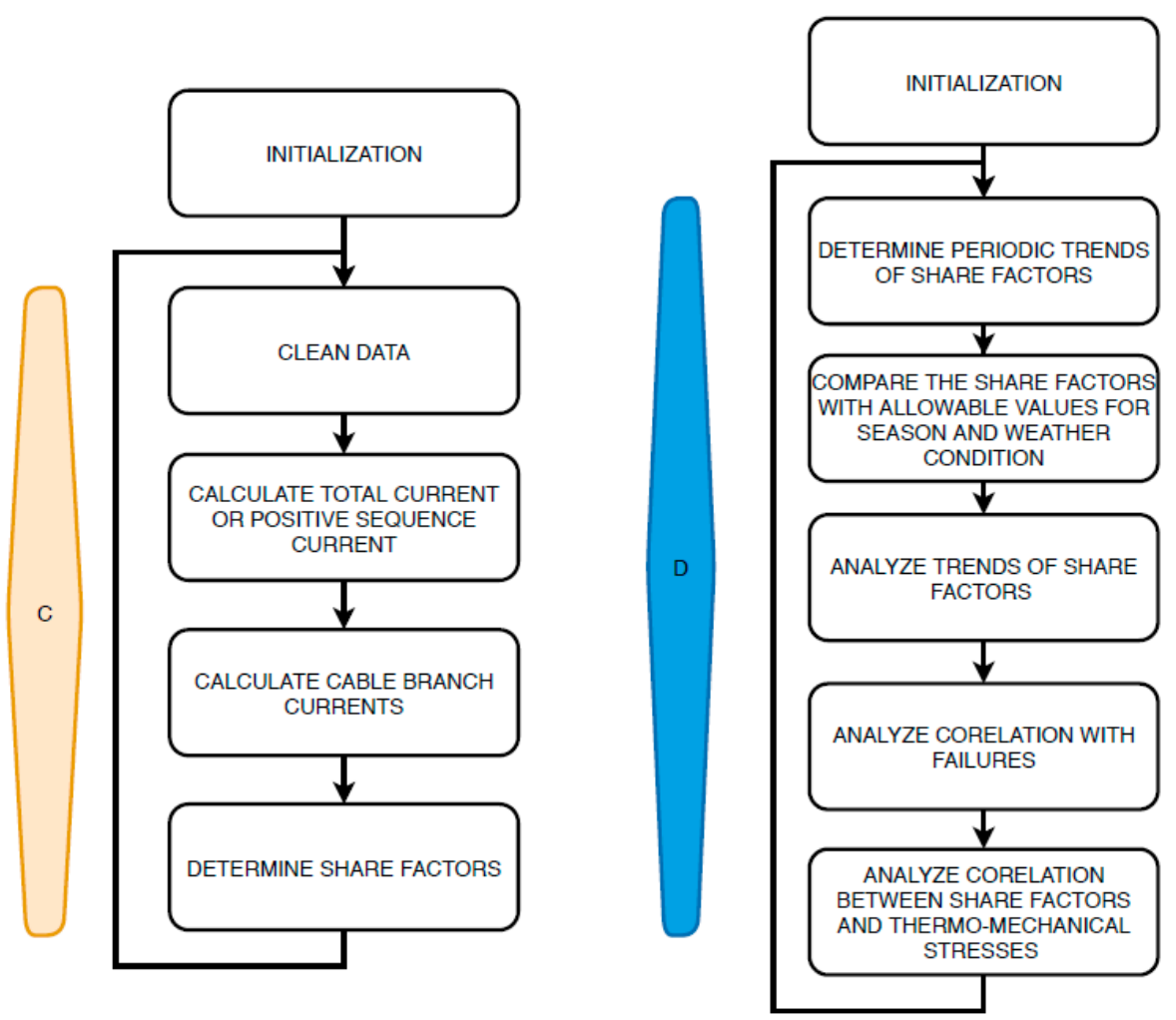

Figure 20. Detailed flowchart presenting data processing and analysis.

AMI meters can be configured to measure both current and power, but in typical applications AMI meters store information only about power flowing through the transformer since power is more convenient to measure energy consumption/production and is also used as an input value for monitoring of the transformer loading [65]. At the same time, however, AMI meters typically record voltages on the low voltage side in order to monitor power quality. Based on the power and voltage one can calculate the current. Data downloaded from AMI are recalculated to obtain the load currents of the cable segments according to Kirchhoff's laws. The exemplary cable feeder is presented in Figure 21. The exemplary feeder consists of three segments-L1, L2, and L3. The current flowing in segment three is equal to load current IL3. The current flowing through segment three is equal to the sum of the currents IL2 and IL3 and so on.

Cleaned data are used to calculate segment share factors (residual return factor) $-R F_{r 110 / 15_{\_} s}$. Share factors are calculated according to set of equations. For the cable line presented in Figure 17, the set of equations takes the form:

$$
\begin{gathered}
I 1_{t 1} \cdot R F_{r 110 / 15 \_s L 1}+I 2_{t 1} \cdot R F_{r 110 / 15 \_s L 2}+I 3_{t 1} \cdot R F_{r 110 / 15_{s} s L 3}=I_{r C S_{-} t 1} \\
I 1_{t 2} \cdot W_{r_{p o w} w_{11}}+I 2_{t 2} \cdot R F_{r 110 / 15_{s} s L 2}+I 3_{t 2} \cdot R F_{r 110 / 15_{-} s L 3}=I_{r C S_{-} t 2} \\
I 1_{t 3} \cdot W_{r_{p o w l}}+I 2_{t 3} \cdot R F_{r 110 / 15 \_s L 2}+I 3_{t 3} \cdot R F_{r 110 / 15 \_s L 3}=I_{r C S_{-} t 3}
\end{gathered}
$$

where $\mathrm{t} 1, \mathrm{t} 2$, and $\mathrm{t} 3$ are following data averaging periods recorded with AMI meters.

The bigger the number of cable segments is, the longer the set of equations are, and therefore, more current averaging periods are needed to solve the set of equations. An alternative possibility to obtain the share factors is to adjust the share factors of the load profiles of the segments in a way which ensures the best fit (the smallest deviation) of the calculated and measured $I_{r c s}$ (averaged and synchronized in the same way as the AMI data). The fitting process is an iterative process, which takes into account boundary conditions obtained from simulation software. The boundary conditions represent the maximum and minimum value of the share factors of the cable segments and are calculated under the assumption that only the analyzed segment is active. For the purpose of the calculation, the lowest 
possible resistances and maximum possible resistances are set as the parameters of the earthing systems (Figure 2). The share factors should be analyzed in a relatively small time window, i.e., 1 to $6 \mathrm{~h}$, to avoid error due to resistance variation, i.e., after heavy rain. The share factors of the cable segments have to be saved in a database for further analysis, i.e., the deviations from long-term trends. The calculated share factors and information about the cable feeder structure are loaded in the initialization process of the data analysis flowchart (Figure 20). The determined share factors are compiled with the total load current and are presented on the $x-y$ plane. Trends describing the share factors as a function of load current are determined and stored in the system for further processing. The share factors are compared with the boundary conditions obtained from the simulation software. The process of calculating the boundary parameters is exactly the same as above; however, seasonal variations or weather conditions of the earthing resistances are considered. The presented approach is similar to fingerprint analysis, which is often used for diagnostic purposes [66,67].

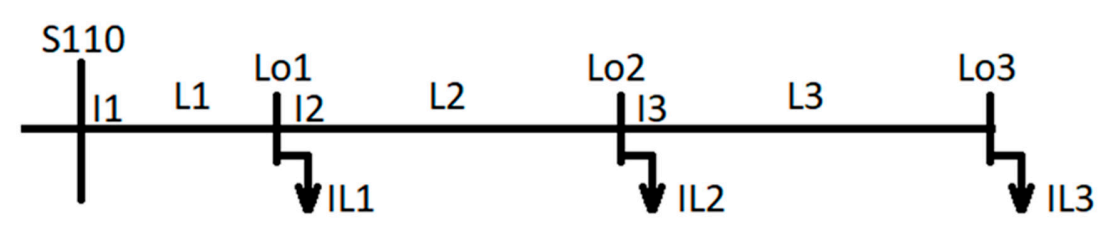

Figure 21. Exemplary load feeder.

Trend lines are further compared with next trend lines obtained for other measuring periods, i.e., trends obtained in 2019 are compared with trends obtained in 2020, in order to find abnormalities, i.e., a shifting trend toward the $\mathrm{x}$ axis. If a significant change of trends is noticed, an alarm is generated. There are a few alarm possibilities-degradation of cable formation, degradation of earthing system, degradation of screen connections, etc. The number of the share factor indicates the location of an abnormal condition, whereas the problem is identified according to conclusions presented in paragraph simulation-if only one share factor is significantly different, one can suspect that the cable screen connection is damaged. In the case of big differences of two trends, one can suspect that degradation of the earthing system between is responsible for the abnormalities. An exceptional case is the last earthing system of the feeder because in that case both earthing system and cable screen degradation may give similar results.

Information about thermo-mechanical stresses is compiled with the share factors. Attention is paid to the relative change of the share factors after the high current incident. Recorded parameters are correlated with the relative change in order to correlate different factors with the cable screen connection degradation. The full functionality of the proposed solutions is achieved when additional data are provided. Data from the failure database can be used to track the causes of the failure.

\subsection{Real-Time Monitoring}

Figure 22 presents the detailed real-time monitoring algorithm. The monitoring is based on analysis of the $I_{r c s}$ amplitude. In order to detect loss of earth continuity, the amplitudes of consecutive measuring periods are subtracted and scaled in \%. If the calculated relative difference is greater than the setting value and the change is not correlated with load variation, the alarm 'Earthing system failure' is activated. The setting value is obtained from the simulation software, after observing the $I_{r c s}$ variation after loss of the earthing system (Sections 4.1 and 4.2). Regardless of the results of the $I_{r c s}$ subtraction, the algorithm is continued and thermo-mechanical stresses are assessed. Monitoring of thermo-mechanical stresses is achieved by analyzing the event register of the digital protection relays. The protection relay has to be configured to store information about high current incidents, i.e., phase to phase faults. Every time the core currents exceed the threshold level, information is stored in the digital register. Afterwards, based on the relation between the cable core currents and the cable screen current, the cable screen current is calculated. Attention is paid to the quantity of thermo-mechanical stresses, maximal current, duration of stresses, and amplitudes of the average currents. The duration of 
the stresses allows the cumulative aging effect to be calculated, which is commonly used for diagnostic purposes [68]. One has to underline that current induced in cable screens under phase to phase fault conditions, in contrast to phase to earth current, does not depend on earthing system resistance. Moreover, the ratio of the cable core current and the screen currents does not depend on fault location. Knowledge about the fault current and the ratio allows the thermo-mechanical stresses to be assessed along the whole cable feeder. However, to assign the stresses to a segment of the line, one has to know the location of a fault. Fortunately, it is relatively simple to assess the location of phase to phase faults, using distance protection algorithms [69]. Thermo-mechanical stresses have a negative degradation impact on cable screen connections; however, it is not clear how strong the effect is, and therefore, further research is needed. It is believed that both laboratory investigation and statistical analysis is needed [70]. The proposed algorithm can be used to pinpoint locations which are subjected to the highest thermo-mechanical stresses, thanks to which attention will be brought to the appropriate places. Afterwards, algorithms analyze the $R F_{r 110 / 15}$ before and after the current incident. If the $R F_{r 110 / 15}$ changes significantly, an alarm is raised.

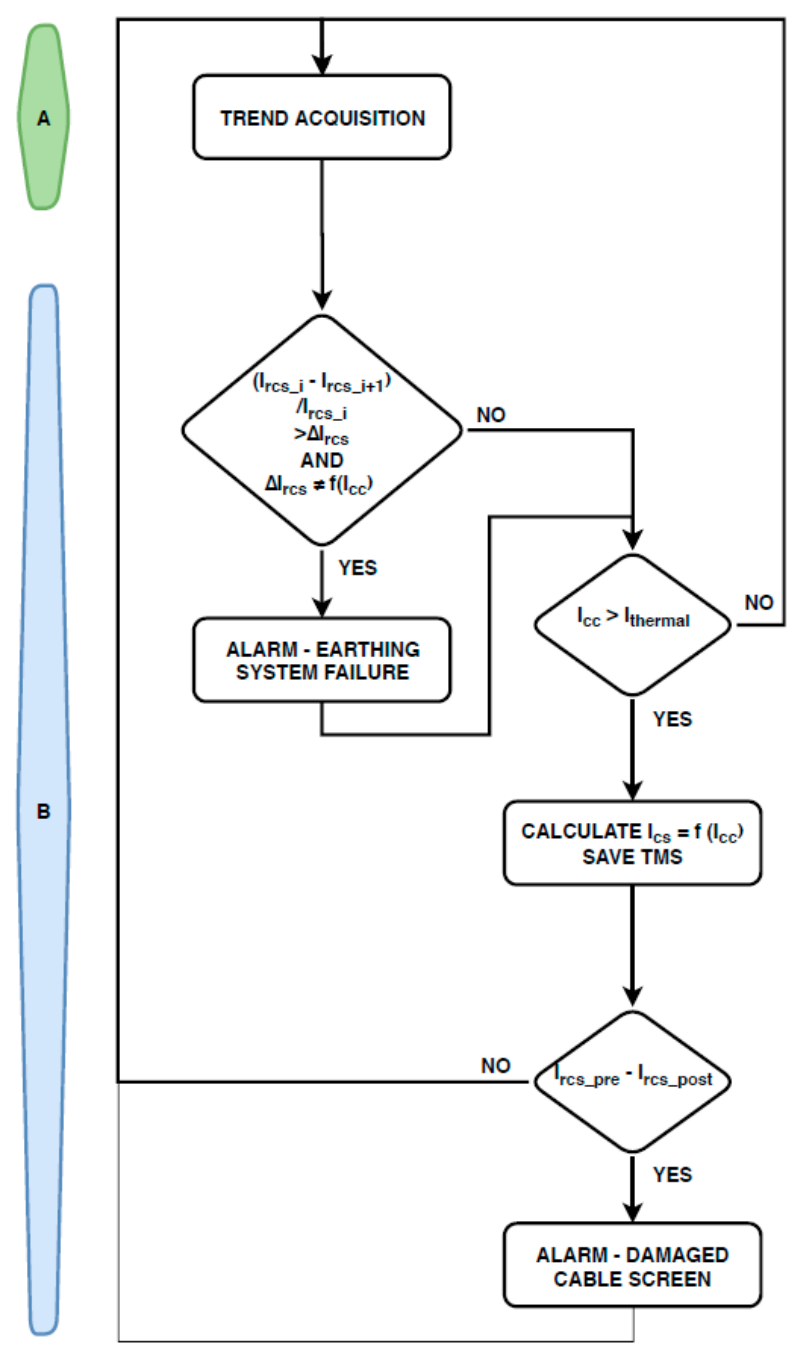

Figure 22. Algorithm for monitoring the thermo-mechanical stresses. $I_{r c s} i / i+1$-consecutive measurements; $\Delta I_{r c s}$-setting of earthing system threshold; $I_{c c}$-load current (phase values); $I_{\text {thremal }}$ - threshold above which the thermomechanical stresses are visible, $I_{\text {rcs_pre/post }}$-current before and after incident under the same loading conditions. Values stored in SAVE TMS (thermo-mechanical stress): time stamp, maximum and average values of phase currents, duration, current, and voltage waveforms (for validation). The block TRENDS ACQUISITION stores average values of total load current and $\mathrm{I}_{\mathrm{rcs}}$ (averaging period is the same as AMI averaging period). 
The proposed thermo-mechanical stress monitoring algorithm is based on current monitoring. The algorithm can be implemented in a conventional power distribution network with just software modifications. The algorithm only indicates lines which were exposed to the greatest thermo-mechanical stresses, however does not give precise information about the stresses. Precise information about the thermo-mechanical stresses can be obtained from strain sensors installed in cable lines.

\section{Discussion}

Monitoring of cable lines is difficult because of the specific cable working conditions:

- cable length can be up to tens of $\mathrm{km}$,

- cable lines are usually buried underground at a depth of about $1 \mathrm{~m}$,

- $\quad$ there are a large amount of cable types and accessories.

MV cable lines are typically inspected in accordance with a time-based maintenance policy. Among different cable line monitoring techniques, only a few solutions have been commercialized or get increased interest of researchers. Among the solutions, one can mention optical fibers for cable temperature measurement, partial discharge measurement, or current monitoring techniques [71]. Installation of optical fibers in cable line allows for precise temperature measurement along the whole cable length, but at the same time is connected with a serious drawback. Installation and repair of the cable line requires two specialists—one cable line specialist and one optical fiber specialist. Partial discharge detection is often presented in the literature as a promising solution for detection of insulation defects [72-74]. Current monitoring techniques are presented in the literature and allow for monitoring of connection continuity, connection flooding, or observe thermal aging of the insulation $[75,76]$. The proposed solutions utilize single point current measurements for monitoring of a few earthing systems and earthing screen connections. The proposed solutions are in the development stage, and further research activities are obviously needed. Unfortunately, due to the coronavirus crisis, research activities are limited due to fear of infection and economic restrictions. The presented solutions have some limitations, i.e., only a few earthing systems can be monitored effectively. It is however noted that a great majority of DSOs use only a time-based maintenance policy for cable line maintenance, without any earthing system monitoring strategy. One has to underline that the number of earthing systems in the distribution system network is very big, and therefore, often only a statistical sample, e.g., $10 \%$ of earthing systems, are investigated. Unfortunately, it is possible that some earthing systems which have not been tested are damaged. It is worth underlining that DSOs have problems with thieves, who steal copper elements of earthing systems, or unreliable contractors, who use iron rods instead of copper or a combination of both-copper rods close to the surface and iron rods underground in order to pass visual inspection and get the work approval [77]. It is noted that monitoring of cable segments may be improved if the return factor $\left(R F_{110 / 15}\right)$, which occurs under phase to earth fault conditions, is analyzed. As is presented in previous papers [16,17], the $R F_{110 / 15}$ depends on cable screen earthing resistance, and therefore, it is possible to use $R F_{110 / 15}$ as a screen earthing system indicator. The concept requires further research.

Effectiveness of the proposed solutions depends on data quality, in particular, results averaging periods of AMI (advanced metering infrastructure). Currently averaged load profiles of MV/LV stations are recorded every $15 \mathrm{~min}$, which is a result of technical constraints, in particular with data transfer limitations and data storage capabilities. It is planned to shorten the results averaging periods to $1 \mathrm{~min}$, which is possible with the current generation of smart meters [78]. Reduction of the averaging period would greatly improve the quality of load profiles and, in turn, would improve the effectiveness of the proposed solutions.

Despite the $I_{r c s}$ monitoring, it is proposed that cable screen currents and thermo-mechanical stresses are monitored. Cable screen currents are assessed based on the ratio of cable screen and core currents. The proposed methods do not give precise information about the thermo-mechanical stresses but indicate lines which were exposed to the greatest thermo-mechanical stresses. Information about 
thermo-mechanical stresses should be compiled with failure data and information data-manufacturer name, cable type, joint type, etc. Data analysis would allow cable lines which are in the worst condition to be indicated. More precise information about the thermo-mechanical stresses can be obtained using mechanical tension sensors, i.e., strain sensors [79]. Unfortunately, not all techniques for mechanical stress measurement can be used for cable line monitoring, as methods which are based on the analysis of magnetic field distribution may give inaccurate results because the magnetic field in the proximity of cable lines may be strongly distorted [80]. Among interesting stress measurement sensors which could be installed in cable lines one can mention capacitive strain sensors or piezoelectric sensors $[81,82]$. Piezoelectric sensors are commercial solutions [83]. Unfortunately, strain sensors do not allow the stresses along the whole cable length to be observed, and therefore, one needs to choose locations for sensor installation which are vulnerable to failures. According to [84], approximately $45 \%$ of failures in cable lines occur in cable joints, and therefore, installation of the stress sensor inside the cable joint would allow many different failures to be detected. Unfortunately, installation of the strain sensors is possible only after the cable screen connection is made, and therefore, it is not possible to install the sensor in the cable factory. As a result, the installation of the cable joint would be more time consuming and would require qualified personnel. Unfortunately, currently it is not clear how to properly install the strain sensors inside different types of cable joints. Furthermore, installation of sensors in cable joints is problematic because the ground is a communication barrier and conventional wireless sensors cannot be used. The sensors for underground infrastructure are in the development stage, an example of which would be the Radio-frequency identification (RFID) underground tag [85]. One has to note that the tags are big and it may be difficult or even impossible to install tags in cable joints. It is also possible to install the electric box in the location of a cable joint; however, that would increase the total cost significantly. Authors believe that strain sensors will not be commonly installed in cable lines however may be used to observe thermo-mechanical stress in laboratory conditions in order to gather more data about the phenomena and improve the effectiveness of the proposed current-based method.

The literature presents a lot of information about structural health monitoring. Structural health sensors could theoretically be used for monitoring of the integrity of the earthing system. An overview of sensors for structural health monitoring is presented in [86]. Structural health sensors are dedicated for the specific type of failure, and therefore, it is difficult to measure the condition of the earthing system effectively because of different failure mechanisms-it is possible that the earthing system will be broken in different locations or that someone will cut off the earthing system or that the earthing system will corrode significantly. The monitoring of the $I_{r c s}$ allows all the failure mechanisms in different locations to be included, whereas structural health sensors allow monitoring of only some failure causes in one location. Moreover, all standards consider the electrical parameters of earthing systems. The electrical parameters can be assessed thanks to the $I_{r c s}$ monitoring, whereas structural health sensors do not provide information about electrical parameters of the earthing systems.

In order to present the proposed solution in a clear manner, a SWOT (Strengths, Weaknesses, Opportunities, and Threats) analysis is made:

Strengths:

- $\quad$ it is able to detect failures which up until now remained undetected,

- $\quad$ it is possible to pre-locate the failure and identify the failure type,

- $\quad$ single point measurement is capable of monitoring a few segments of cable line,

- the proposed solution can be integrated with an existing diagnostic system, i.e., the SORAL system [87], which greatly reduces the workload for implementation,

- $\quad$ additional sensors could be installed in depths of the network.

Weaknesses:

- the effectiveness of the system is reduced when the distance from measurement location increases,

- residual earth fault current is observed in two point earthed cable lines only, therefore, the solutions cannot be used in one point grounded lines [88], 
- further research is needed, particularly in locations of industrial plants and traction loads, which could be a source of stray currents.

Opportunities:

- $\quad$ the system could be used for monitoring of high voltage (HV) lines,

- $\quad$ it is believed that the functionality of the system can be improved, i.e., by analyzing $R F_{110 / 15}$ under phase to ground fault conditions,

- the system is able to detect any abnormal current rise and therefore can protect cable screens from overheating,

- the cost of sensors installed in the depths of the network could be reduced if current sensors were integrated with other smart city sensors, e.g., weather stations, cameras, etc.

Threats:

- erroneous data about cable lines could compromise the effectiveness of the system.

\section{Summary}

The contribution of the paper is connected with the development of new solutions for the monitoring of earthing systems of cables lines and cable screen connections, i.e., cable joints. The monitoring of the distribution feeders built out of one cable segment is validated using measurements in an MV network. The proposed solution is also able to monitor complex, multi-segment cable lines if AMI data are provided. Moreover, it is possible to pre-locate the failures of cable line earthing systems or screen connections. The principle of operation of the proposed solution is based on analysis of trends and observing criterion values-residual cable screen earthing current and shares of cable segments in 110/15 residual screen earthing current. Additionally, a tool for monitoring of thermo-mechanical stresses in cable screens is presented. The tool uses information obtained from conventional digital protection relays (short circuit, average and peak currents, and duration of the short circuit) and the transformation ratio between cable cores and screens currents to calculate the thermo-mechanical stresses in cable screens and in cable joints. Different failure scenarios are analyzed in PowerFactory software, and characteristic features of failures are outlined.

Further research is focused on analyzing the effectiveness of the system in complex cable feeders. Analysis of other cable screen current sources is also planned, in particular stray currents; therefore, taking measurements in lines which supply industrial and traction loads is planned. The goal of the research is to develop an expert system that can recognize the origination of current sources and is able to extract useful information automatically. Attention will be paid to the whole frequency spectrum, which is often used for diagnostic purposes [89].

Author Contributions: Conceptualization, K.L.; methodology, K.L.; software, K.L.; validation, J.L., A.T., Z.N., K.L., and J.Z.; formal analysis, A.T. and Z.N.; investigation, K.L.; resources, Z.N. and J.Z.; data curation, K.L.; writing-original draft preparation, K.L.; writing—review and editing, K.L. and A.T.; visualization, K.L.; supervision, A.T. and Z.N. project administration, J.L.; funding acquisition, Z.N. All authors have read and agreed to the published version of the manuscript.

Funding: The research was financed from resources of the Ministry of Science and Higher Education for Statutory Activities No. 0711/SBAD/4410, name of the task: Improvement of Reliability of Supply in Distribution Network.

Conflicts of Interest: The authors declare no conflicts of interest.

\section{References}

1. Bargiel, J.; Goc, W.; Sowa, P.; Teichman, B. Niezawodność zasilania odbiorców z sieci średniego napięcia. In Proceedings of the Materiały XVI Konferencji Naukowo-Technicznej Rynek Energii Elektrycznej, Kazimierz Dolny, Poland, 12-15 May 2010.

2. Qamar, A.; Umair, M.; Yang, F.; Uzair, M.; Kaleem, Z. Derivative Method Based Orientation Detection of Substation Grounding Grid. Energies 2018, 11, 1873. [CrossRef] 
3. Li, X.; Yang, F.; Ming, J.; Jadoon, A.; Han, S. Imaging the Corrosion in Grounding Grid Branch with Inner-Source Electrical Impedance Tomography. Energies 2018, 11, 1739. [CrossRef]

4. Yang, F.; Wang, Y.; Dong, M.; Kou, X.; Yao, D.; Li, X.; Gao, B.; Ullah, I. A Cycle Voltage Measurement Method and Application in Grounding Grids Fault Location. Energies 2017, 10, 1929. [CrossRef]

5. Wang, X.; Fu, Z.; Wang, Y.; Liu, R.; Chen, L. A Non-Destructive Testing Method for Fault Detection of Substation Grounding Grids. Sensors 2019, 19, 2046. [CrossRef]

6. Perng, J.W.; Kuo, Y.C.; Lu, S.P. Grounding System Cost Analysis Using Optimization Algorithms. Energies 2018, 11, 3484. [CrossRef]

7. Vycital, V.; Ptacek, M.; Topolanek, D.; Toman, P. On Minimisation of Earthing System Touch Voltages. Energies 2019, 12, 3838. [CrossRef]

8. Campoccia, A.; Sanseverino, E.R.; Zizzo, G. Analysis of interconnected earthing systems of MV/LV substations in urban areas. In Proceedings of the Universities Power Engineering Conference, Padova, Italy, 1-4 August 2008.

9. Long, X.; Dong, M.; Xu, W.; Li, Y.W. Online Monitoring of Substation Grounding Grid Conditions Using Touch and Step Voltage Sensors. IEEE Trans. Smart Grid 2012, 3, 761-769. [CrossRef]

10. ETS Cable Component, Characteristics of A Good Earthing System. Available online: https://www. etscablecomponents.com/2015/08/354/ (accessed on 1 May 2020).

11. Eneida, Earthing System Monitoring and Optimization. Available online: https://eneida.io/earth/ (accessed on 1 May 2020).

12. Parmar, J. Earthing in Electrical Network. 2011. Available online: https://electrical-engineering-portal.com/ earthing-in-electrical-network-purpose-methods-and-measurement (accessed on 1 May 2020).

13. PTPiREE. Energetyka Dystrybucja i Przesyt; PTPiREE: Poznan, Poland, 2017.

14. ServersCheck BV®, Ground (Earthing) Monitoring Sensor. Available online: https://serverscheck.com/ sensors/sensor_grounding.asp (accessed on 1 May 2020).

15. Stahl, Ground Monitoring Devices. Available online: https://r-stahl.com/en/global/products/automationinterfaces-and-solutions/grounding-monitoring-devices-and-systems/grounding-monitoring-devices/ (accessed on 1 May 2020).

16. Lowczowski, K.; Lorenc, J.; Zawodniak, J.; Dombek, G. Detection and Location of Earth Fault in MV Feeders Using Screen Earthing Current Measurements. Energies 2020, 13, 1293. [CrossRef]

17. Lowczowski, K.; Lorenc, J.; Andruszkiewicz, J.; Nadolny, Z.; Zawodniak, J. Novel Earth Fault Protection Algorithm Based on MV Cable Screen Zero Sequence Current Filter. Energies 2019, 12, 3190. [CrossRef]

18. Lowczowski, K.; Nadolny, Z.; Olejnik, B. Analysis of Cable Screen Currents for Diagnostics Purposes. Energies 2019, 12, 1348. [CrossRef]

19. Gulski, E.; Wester, F.J.; Boone, W.; Schail, N.V.; Steennis, E.; Groot, E.; Pellis, J.; Grotenhuis, B. Knowledge Rules Support for CBM of Power Cable Circuits. In Proceedings of the CIGRE Session, Paris, France, 25-30 August 2002.

20. Szymenderski, J.; Machczynski, W.; Budnik, K. Modeling Effects of Stochastic Stray Currents from D.C. Traction on Corrosion Hazard of Buried Pipelines. Energies 2019, 12, 4570. [CrossRef]

21. JWG B3.35/CIRED. Substation Earthing System Design Optimization through the Application of Quantified Risk Analysis; CIGRE: Paris, France, 2018.

22. Arcadius Tokognon, C., Jr.; Gao, B.; Tian, G.Y.; Yan, Y. Structural Health Monitoring Framework Based on Internet of Things: A Survey. IEEE Internet Things J. 2017, 4, 619-635. [CrossRef]

23. Huang, Q.; Jing, S.; Yi, J.; Zhen, W. Innovative Testing and Measurement Solutions for Smart Grid; Wiley: Singapore, 2015.

24. NCD, IoT Long Range Wireless AC Current Monitor. Available online: https://store.ncd.io/product/wirelessac-current-monitor/ (accessed on 14 May 2020).

25. Holger, K.; Willig, A. Protocols and Architectures for Wireless Sensor Networks; John Wiley \& Sons: Chichester, UK, 2007.

26. Beanair, BeanDevice $® 2.4 \mathrm{GHz}$ One-T. Available online: https://www.beanair.com/wireless-iot-temperaturesensors-overview.html (accessed on 25 May 2020).

27. Harid, N.; Bogias, A.C.; Griffiths, H.; Robson, S.; Haddad, A. A Wireless System for Monitoring Leakage Current in Electrical Substation Equipment. IEEE Access 2016, 4, 2695-2975. [CrossRef] 
28. Tang, L.; Lu, Z.; Fan, B. Energy Efficient and Reliable Routing Algorithm for Wireless Sensors Networks. Appl. Sci. 2020, 10, 1885. [CrossRef]

29. Inhand, Overhead Line Sensor. 2020. Available online: https://www.inhandnetworks.com/solutions/IWOS/ overhead-line-sensor.html (accessed on 20 May 2020).

30. Adeunis, Current Sensor: Curren Measurement. 2020. Available online: https://www.adeunis.com/en/ produit/current-sensor-current-measurement/ (accessed on 20 May 2020).

31. Wang, Y.; Chen, Q.; Hong, T.; Kang, C. Review of Smart Meter Data Analytics: Applications, Methodologies, and Challenges. IEEE Trans. Smart Grid 2019, 10, 3125-3148. [CrossRef]

32. Mohassel, R.R.; Fung, A.; Mohammadi, F.; Raahemifar, K. A survey on Advanced Metering Infrastructure. Int. J. Electr. Power Energy Syst. 2014, 63, 473-484. [CrossRef]

33. Andreadou, N.; Kotsakis, E.; Masera, M. Smart Meter Traffic in a Real LV Distribution Network. Energies 2018, 11, 1156. [CrossRef]

34. Teng, J.H.; Chao, C.W.; Liu, B.H.; Huang, W.H.; Chiu, J.C. Communication Performance Assessment for Advanced Metering Infrastructure. Energies 2019, 12, 88. [CrossRef]

35. Canha, L.N.; Ramos, L.F.; Sen, P.K.; Carvalho, R.S. Communication System Design for an Advanced Metering Infrastructure. In Proceedings of the IEEE International Conference on Smart Energy Grid Engineering (SEGE), Oshawa, ON, Canada, 12-15 August 2018.

36. Szulim, R.; Michta, E.; Markowski, A. Communication structures and rule based energy management in AMI systems. Prz. Elektrotechniczny 2014, 9, 2014.

37. Huang, X.; Hu, T.; Ye, C.; Xu, G.; Wang, X.; Chen, L. Electric Load Data Compression and Classification Based on Deep Stacked Auto-Encoders. Energies 2019, 12, 653. [CrossRef]

38. Bonetto, R.; Bui, N.; Lakkundi, V.; Olivereau, A.; Serbanati, A.; Rossi, M. Secure communication for smart IoT objects: Protocol stacks, use cases and practical examples. In Proceedings of the IEEE International Symposium on a World of Wireless, Mobile and Multimedia Networks (WoWMoM), San Francisco, CA, USA, 25-28 June 2012.

39. Thompson, G. Validating Field Performance of AMI Systems. Electr. Energy T D Mag. 2005, 6, 30-35.

40. Enea Operator. Stacje Transformatorowe Kompaktowe Prefabrykowane SN/nn Oraz Złacza/Szafy Kablowe SN; Enea Operator: Poznan, Poland, 2015.

41. Schneider Electric, Discover the PowerLogic ${ }^{\mathrm{TM}}$ PowerTag Energy Sensor. Available online: https://www.se. com/ww/en/work/products/product-launch/powertag/ (accessed on 12 May 2020).

42. Montes Figueroa, J.M.; Pinilla Rodriguez, J.; Ramos, F. IOT-PMU. How to Improve Observability on the Distribution Networks. In Proceedings of the 25th International Conference on Electricity Distribution, Madrid, Spain, 3-6 June 2019.

43. Presac, Current Monitoring Sensors. 2020. Available online: https://www.pressac.com/current-monitoringsensors/ (accessed on 10 June 2020).

44. WG B3.44, Survey. 2017. Available online: https://www.surveymonkey.de/r/33V622Y (accessed on 15 May 2020).

45. Habrych, M.; Wiśniewski, G.; Miedzinski, B.; Lisowiec, A.; Fjalkowski, Z. HDI PCB Rogowski coils for automated electrical power system applications. IEEE Trans. Power Deliv. 2017, 33, 1536-1544. [CrossRef]

46. Habrych, M.; Wisniewski, G.; Miedzinski, B.; Lisowiec, A. Applicability of Rogowski coils made in PCB HDI technology in power system protections. In Proceedings of the Modern Electric Power Systems, Wroclaw, Poland, 6-8 September 2015.

47. Norouzi, A. Open Phase Conditions in Transformers Analysis and Protection Algorithm. In Proceedings of the 66th Annual Conference for Protective Relay Engineers, College Station, TX, USA, 8-11 April 2013.

48. Megger, Fault Finding Solutions. Section II-Fault Locating Procedure. In Powerpoint Engineering; Megger: Kent, UK, 2003.

49. Kaushik, M. Single Phasing in Electrical Motors: Causes, Effects, and Protection Methods. In Marine Electrical; Marine Insight. 2019. Available online: https://www.marineinsight.com/marine-electrical/single-phasing-inelectrical-motors-causes-effects-and-protection-methods/ (accessed on 10 May 2020).

50. IEEE Std 998-2012. IEEE Guide for the Protection of Communication Installations from Lightning Effects; IEE: New York, NY, USA, 2012.

51. Kumar, R.; Kumar, P. Modelling of Stray-Load Loss for Medium Power Induction Motors. In Proceedings of the IECON 2018 - 44th Annual Conference of the IEEE Industrial Electronics Society, Washington, DC, USA, 21-23 October 2018. 
52. Wang, X.H.; Song, Y.H.; Jung, C.K.; Lee, J.B. Tackling sheath problems: Latest research developments in solving operational sheath problems in underground power transmission cables. Electr. Power Syst. Res. 2007, 77, 1449-1457. [CrossRef]

53. DigSilent, Quasi-Dynamic Simulation. 2020. Available online: https://www.digsilent.de/en/quasi-dynamicsimulation.html (accessed on 1 June 2020).

54. Lowczowski, K. Badanie wpływu ułożenia kabli na straty energii w żyle powrotnej-Symulacja w programie PowerFactory. Prz. Elektrotechniczny 2016, 10, 54-57. [CrossRef]

55. Sowa, P.; Kumala, R.; Luszcz, K. Modeling of power system components during electromagnetic transients. Int. J. Innov. Sci. Eng. Technol. 2014, 1, 715-719.

56. IEC 60287-1-1 Electric Cables-Calculation of the Current Rating Part 1: Current Rating Equations (100\% Load Factor) and Calculation of Losses Section 1: General; International Electrotechnical Commission: Geneva, Switzerland, 2006.

57. Enea Operator. Elektroenergetyczne Linie Kablowe Średniego Napięcia; Enea Operator: Poznan, Poland, 2019.

58. GMCiUK, GMC-I Prosys ACP 3003-3/24. Available online: https://www.gmciuk.com/gmc_i-prosys-acp3003_3 \{\}24-329 (accessed on 18 May 2020).

59. Gielniak, J.; Graczkowski, A.; Moranda, H.; Przybylek, P.; Walczak, K.; Nadolny, Z.; Moscicka-Grzesiak, H.; Feser, K.; Gubanski, S.M. Moisture in cellulose insulation of power transformers-Statistics. IEEE Trans. Dielectr. Electr. Insul. 2013, 20, 982-987. [CrossRef]

60. Bishop, C.M. Pattern Recognition and Machine Learning; Springer: Berlin/Heidelberg, Germany, 2006.

61. Tauron Dystrybucja. Standard Techniczny nr 11/2015 Budowy Układów Uziomowych w Sieci Dystrybucyjnej TAURON Dystrybucja, S.A.; Tauron Dystrybucja: Krakow, Poland, 2017.

62. Musial, E. Uziomy Fundamentowe i Parafundamentowe. INPE 2011, 143, 3-33.

63. King, L. The Common Information Model for Distribution; EPRI: Palo Alto, CA, USA, 2008.

64. IEEE PES PSRC C5. Considerations for Use of Disturbance Recorders; IEEE Power System Relaying and Control Committee, 2006.

65. IEEE C57.91. IEEE Guide for Loading Mineral-Oil-Immersed Transformers and Step-Voltage Regulators; IEEE Power and Energy Society: New York, NY, USA, 2012.

66. Moranda, H.; Sztolcman, M.; Walczak, K.; Moscicka-Grzesiak, H. Enlargement of fingerprint in procedure of defects recognition in oil-paper insulation using time parameters of PD. In Proceedings of the XIV International Symposium on High Voltage Engineering, Beijing, China, 25-29 August 2005.

67. Sikorski, W. Development of Acoustic Emission Sensor Optimized for Partial Discharge Monitoring in Power Transformers. Sensors 2019, 19, 1865. [CrossRef]

68. Sikorski, W.; Walczak, K. Analiza trendu parametrow wyładowan niezupelnych realizowana przez system monitoringu transformatorow energetycznych Pdtracker. Prz. Elektrotechniczny 2014, 10, 168-171.

69. Campos, J.T.L.S.; Sanca, H.S.; Costa, F.B.; Souza, B.A. Distance Protection Analysis Applied for Distribution System with Distributed Generation. Prz. Elektrotechniczny 2018, 3, 13-17. [CrossRef]

70. Moranda, H. Prognozowanie zawilgocenia izolacji celulozowej transformatorów na postawie wyników analizy statystycznej danych pomiarowych. Program, Ocena zawilgocenia transformatorów". Prz. Elektrotechniczny 2010, 11, 162-165.

71. Wojtas, S.; Zawodniak, J. Aspekty praktyczne wykonywania uziemień stanowisk słupowych z głowicami kablowymi. Autom. Elektr. Zaklocenia 2015, 6, 42-54.

72. NKT Photonics, LISO EN.SURE Sensor Cables. 2020. Available online: https://www.nktphotonics.com/lios/ en/product/lios-en-sure-sensor-cables/ (accessed on 18 May 2020).

73. Tian, Y.; Lewin, P.; Wilkinson, J.S.; Schroder, G.; Sutton, S.J.; Swingler, S.G. An improved optically based PD detection system for continuous on-line monitoring of HV cables. IEEE Trans. Dielectr. Electr. Insul. 2006, 12, 1222-1234. [CrossRef]

74. Noske, S. Measurement of Partial Discharges in Medium Voltage Cable Lines. Acta Energetica 2009, 1, $105-114$.

75. Zhou, C.; Song, X.; Michel, M.; Hepburn, D.M. On-line partial discharge monitoring in medium voltage underground cables. IET Sci. Meas. Technol. 2009, 3, 354-363. [CrossRef]

76. Han, Y.J.; Lee, H.M.; Shin, Y.J. Thermal aging estimation with load cycle and thermal transients for XLPE-insulated underground cable. In Proceedings of the IEEE Conference on Electrical Insulation and Dielectric Phenomenon (CEIDP), Fort Worth, TX, USA, 22-25 October2017. 
77. Yang, Y.; Hepburn, D.M.; Zhou, C.; Zhou, W.; Jiang, W.; Tian, Z. On-line monitoring and analysis of the dielectric loss in cross-bonded HV cable system. Electr. Power Syst. Res. 2017, 149, 89-101. [CrossRef]

78. LandisGyr. Landis+Gyr S650 Smart Grid Terminal; LandisGyr: Zug, Switzerland, 2012.

79. Baumer, Strain Sensors. 2020. Available online: https://www.baumer.com/dk/en/product-overview/forcesensors-strain-sensors/strain-sensors/c/311 (accessed on 4 June 2020).

80. Wilson, J.; Barrans, S. Residual magnetic field sensing for stress measurement. Sens. Actuators A Phys. 2007, 135, 381-387. [CrossRef]

81. Shkel, Y.M.; Lee, H.Y.; Peng, Y. Dielectrostrictive Sensor for Measuring Deformation. U.S. Patent No. 7,726,199 B2, 8 October 2009.

82. Dagdeviren, C.; Joe, P.; Tuzman, O.L.; Park, K.I.; Lee, K.J.; Shi, Y.; Huang, Y.; Rogers, J.A. Recent progress in flexible and stretchable piezoelectric devices for mechanical energy harvesting, sensing and actuation. Extrem. Mech. Lett. 2016, 9, 269-281. [CrossRef]

83. Hammerschmidt, C. Sensor Detects Mechanical Stress in Wind Turbines. 2014. Available online: https: //www.eenewseurope.com/news/sensor-detects-mechanical-stress-wind-turbines (accessed on 1 June 2020).

84. Ravish, P.Y. Application of Statistical Life Data Analysis for Cable Joints in MV Distribution Networks. Master's Thesis, Delft University of Technology, Delft, The Netherlands, 2010.

85. Abdelnour, A.; Lazaro, A.; Villarino, R.; Kaddour, D.; Tedjini, S.; Girbau, D. Passive Harmonic RFID System for Buried Assets Localization. Sensors 2018, 18, 3635. [CrossRef]

86. Zhang, J.; Tian, G.Y.; Marindra, A.M.J.; Sunny, A.I.; Zhao, A.B. A Review of Passive RFID Tag Antenna-Based Sensors and Systems for Structural Health Monitoring Applications. Sensors 2017, 17, 265. [CrossRef]

87. Noske, S.; Grzelka, S.; Kołodziejczyk, K. SORAL-wszystko co chciałbys wiedziec o swojej sieci kablowej. In Proceedings of the XVIII Konferencja Systemy Informatyczne w Energetyce SIwE, Wisla, Poland, 26-29 November 2019.

88. Schott-Szymczak, A.; Walczak, K. Analysis of Overvoltages Appearing in One-Sidedly Ungrounded MV Power Cable Screen. Energies 2020, 13, 1821. [CrossRef]

89. Gielniak, J.; Graczkowski, A.; Moranda, H.; Przybylek, P.; Walczak, K.; Moscicka-Grzesiak, H. Patterns of Moisture Content in Insulation of Paper and Synthetic Ester Midel 7131 using the FDS Technique. In Proceedings of the XVII International Symposium on High Voltage Engineering, Hannover, Germany, 22-26 August 2011.

(C) 2020 by the authors. Licensee MDPI, Basel, Switzerland. This article is an open access article distributed under the terms and conditions of the Creative Commons Attribution (CC BY) license (http://creativecommons.org/licenses/by/4.0/). 